\title{
A GABAergic neural circuit in the ventromedial hypothalamus mediates chronic stress-induced bone loss
}

\author{
Fan Yang, ${ }^{1,2,3,4,5}$ Yunhui Liu, ${ }^{1,2,3,4,5}$ Shanping Chen, ${ }^{1,2,3,4,5}$ Zhongquan Dai, ${ }^{6}$ Dazhi Yang, ${ }^{7}$ Dashuang Gao, ${ }^{1,2,3,4,5}$ Jie Shao, ${ }^{1,2,3,4,5}$ \\ Yuyao Wang, ${ }^{1,2,3,4}$ Ting Wang, ${ }^{1,2,3,4}$ Zhijian Zhang, ${ }^{8,9}$ Lu Zhang, ${ }^{10}$ William W. Lu, ${ }^{10}$ Yinghui Li, ${ }^{6}$ and Liping Wang ${ }^{1,2,3,4,5}$ \\ 'Brain Cognition and Brain Disease Institute, Shenzhen Institutes of Advanced Technology, Chinese Academy of Sciences (CAS), ${ }^{2}$ CAS Key Laboratory of Brain Connectome and Manipulation, ${ }^{3}$ Cuangdong \\ Provincial Key Laboratory of Brain Connectome and Behavior, ${ }^{4}$ Shenzhen-Hong Kong Institute of Brain Science-Shenzhen Fundamental Research Institutions, Shenzhen, China. ${ }^{5}$ University of Chinese \\ Academy of Sciences, Beijing, China. ${ }^{6}$ State Key Laboratory of Space Medicine Fundamentals and Application, China Astronaut Research and Training Center, Beijing, China. ${ }^{7}$ Department of Orthopedics, \\ Union Shenzhen Hospital, Huazhong University of Science and Technology, Shenzhen, China. ${ }^{8}$ Center for Brain Science, Key Laboratory of Magnetic Resonance in Biological Systems and State Key Laboratory \\ of Magnetic Resonance and Atomic and Molecular Physics, Wuhan Institute of Physics and Mathematics, CAS, Wuhan, China. ${ }^{9}$ Center for Excellence in Brain Science and Intelligence Technology, CAS, \\ Shanghai, China. ${ }^{10}$ Department of Orthopaedics and Traumatology, The University of Hong Kong, Hong Kong, China.
}

\begin{abstract}
Homeostasis of bone metabolism is regulated by the central nervous system, and mood disorders such as anxiety are associated with bone metabolism abnormalities, yet our understanding of the central neural circuits regulating bone metabolism is limited. Here, we demonstrate that chronic stress in crewmembers resulted in decreased bone density and elevated anxiety in an isolated habitat mimicking a space station. We then used a mouse model to demonstrate that GABAergic neural circuitry in the ventromedial hypothalamus (VMH) mediates chronic stress-induced bone loss. We show that CABAergic inputs in the dorsomedial VMH arise from a specific group of somatostatin neurons in the posterior region of the bed nucleus of the stria terminalis, which is indispensable for stress-induced bone loss and is able to trigger bone loss in the absence of stressors. In addition, the sympathetic system and glutamatergic neurons in the nucleus tractus solitarius were employed to regulate stress-induced bone loss. Our study has therefore identified the central neural mechanism by which chronic stress-induced mood disorders, such as anxiety, influence bone metabolism.
\end{abstract}

\section{Introduction}

Mood disorders, such as depression and anxiety, are closely related to bone metabolism abnormalities (1-3) and can lead to low bone mass and increased risk of fracture $(4,5)$. Another well-established cause of bone loss is microgravity during spaceflight (6). However, decreased bone formation in astronauts can occur $(7,8)$ or progressively deteriorate (9) under normal gravity following space missions. Chronic stressors in extreme microenvironments may not only induce psychological changes in crewmembers but also continuously disturb peripheral metabolism through the central nervous system (CNS) $(10,11)$. Based on these findings, we reasoned that stress-induced psychological disorders, such as anxiety, might directly contribute to bone loss through the CNS. However, the exact neural mechanism behind chronic stress-induced bone loss is not clear.

Bone metabolism is a dynamic physiological process that is finely regulated by the brain (12). Central signals from the brain exert an important influence on bone metabolism (13-15). For example, leptin signaling in the hypothalamus inhibits bone for-

Authorship note: FY and Y. Liu are co-first authors.

Conflict of interest: The authors have declared that no conflict of interest exists. Copyright: (c) 2020, American Society for Clinical Investigation.

Submitted: January 3, 2020; Accepted: August 26, 2020; Published: November 9, 2020

Reference information: J Clin Invest. 2020;130(12):6539-6554.

https://doi.org/10.1172/JCl136105. mation through the sympathetic nervous system (14), and orexin, which is produced in the hypothalamus, is a critical regulator of skeletal homeostasis and exerts regulation of bone mass (16). The hypothalamus is an important neural control center relaying information from higher centers in the forebrain to regulate body metabolism (17). The ventromedial hypothalamus (VMH), one of many hypothalamic nuclei, is a distinct region important in regulating emotion $(18,19)$, energy balance $(13,20)$, and bone metabolism $(15,21)$. Knocking out SF1 neurons, which are present in the VMH, results in significantly increased anxiety-like behaviors in mice (22), and optogenetic activation of dorsal VMH promotes a variety of context-dependent defense-like and autonomic responses (23). Moreover, inactivation of CaMKK $\beta$ in SF1-expressing neurons in the $\mathrm{VMH}$ results in a severe low-bone phenotype (21), while serotoninergic projections from the raphe nucleus to the VMH may regulate SF1 neurons via Htr2c receptors to promote bone accrual (15). Manipulation of the transcription factor AP1 in VMH SF1-expressing neurons leads to an increase in energy and a decrease in bone density, suggesting that the $\mathrm{VMH}$ regulates bone homeostasis and energy metabolism through disparate neural pathways (24). Collectively, these findings suggest that differential neural pathways direct VMH neurons to regulate emotions and bone metabolism in a top-down manner. However, it is not known how the specific neural circuit involving the VMH integrates stress information and regulates downstream bone metabolism. 
A

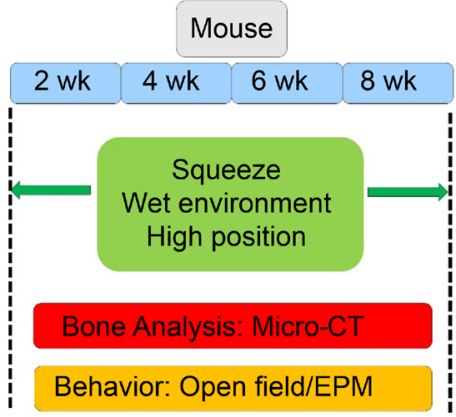

B
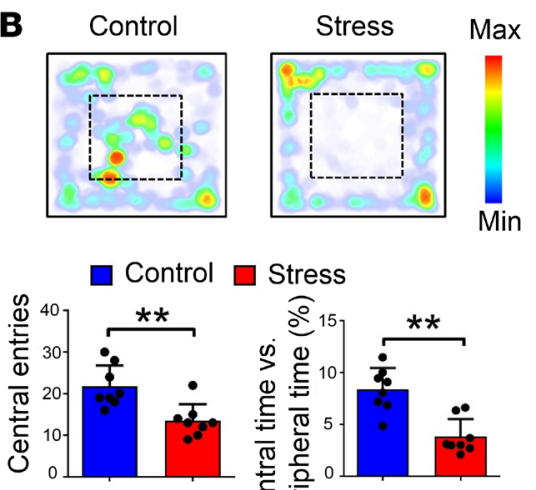

\section{Stress}

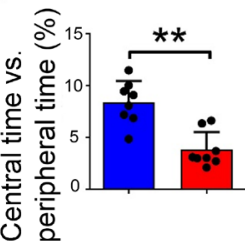

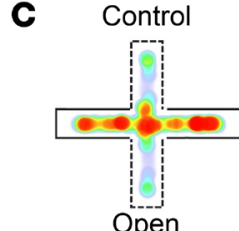

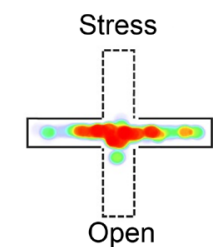

Control $\square$ Stress

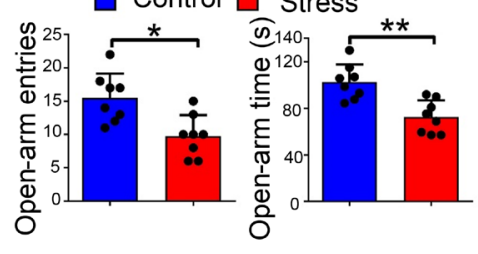

D
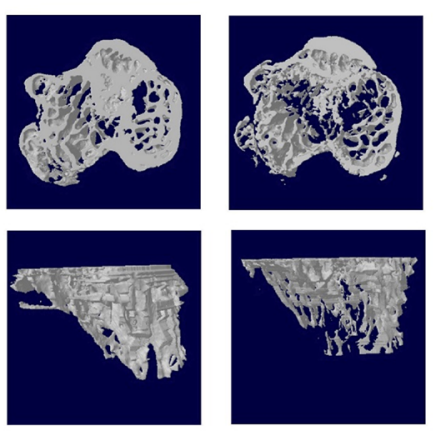

E
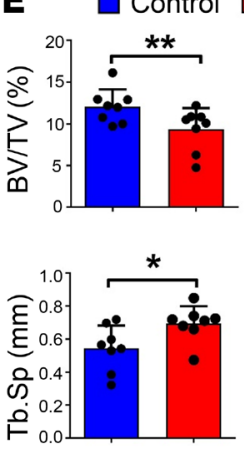
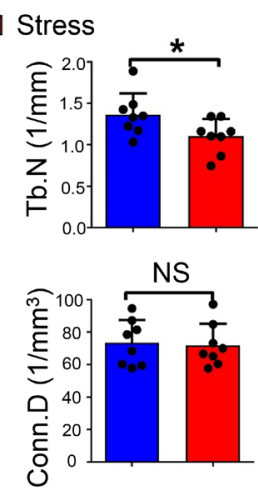

$\mathbf{F}$

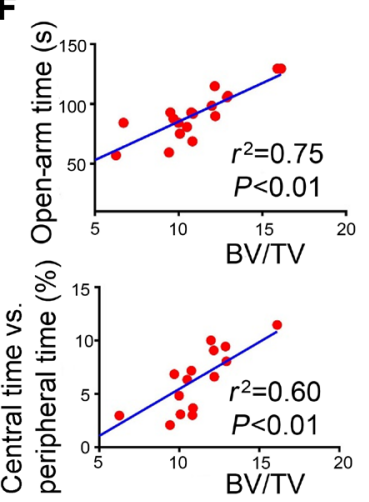

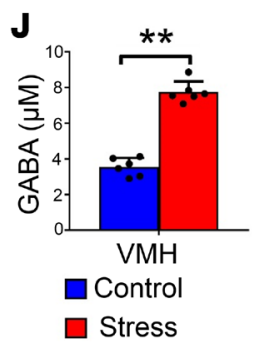

G

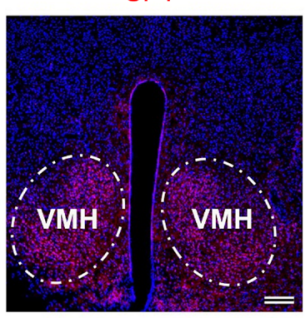

K

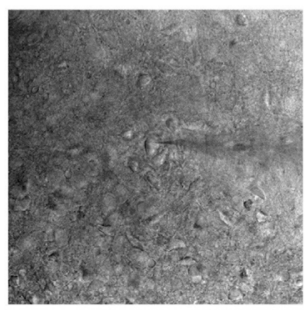

H

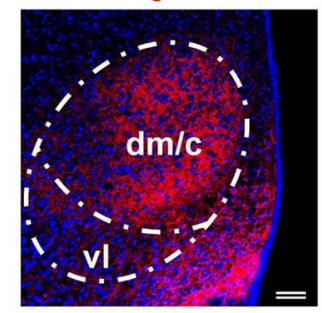

Control

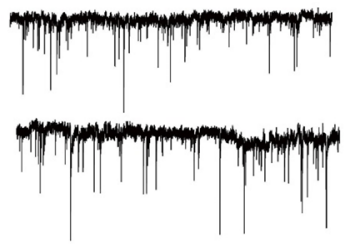

I

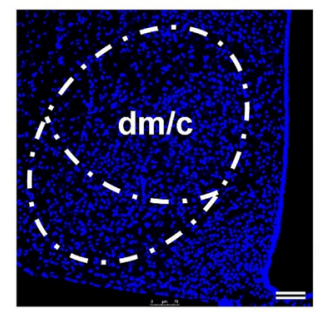

GAD65

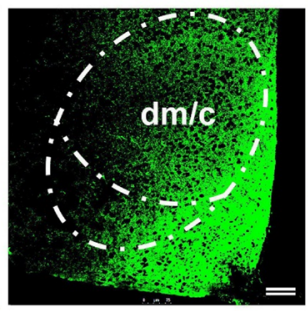

Merge

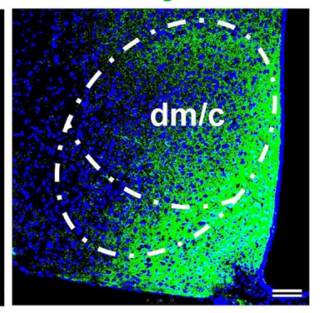

Stress

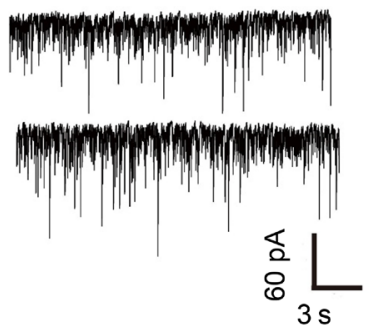

$\mathbf{L}$

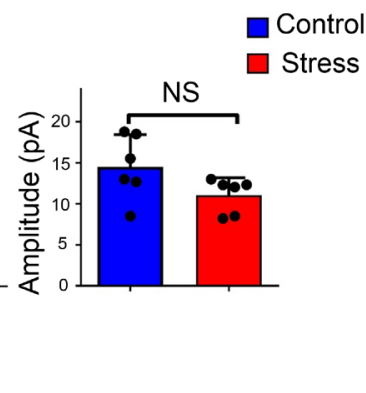

Figure 1. A mouse model of chronic stress-induced anxiety-like behavior and bone loss. (A) Schematic showing generation of the unpredictable chronic mild stress-induced anxiety mouse model. (B) Open-field (OF) test comparing the stress and control groups. Mice in the stress group exhibited significantly fewer entries to the central area and spent less time exploring the central area. Values represent mean $\pm \operatorname{SD}\left(n=8\right.$ per group; ${ }^{* *} P<0.01$; Student's $t$ test). (C) Elevated plus maze (EPM) test comparing stress and control groups. Mice in the stress group exhibited significantly fewer entries to the open arms and spent less time there. Values represent mean $\pm S D\left(n=8\right.$ per group; ${ }^{*} P<0.05$, ${ }^{* *} P<0.01$; Student's $t$ test). (D) Representative coronal (top) and sagittal (bottom) plane images of proximal tibia. Mice in the stress group displayed a low-bone mass phenotype. (E) Micro-CT analysis of trabecular bone volume/tissue volume (BV/TV), trabecular number (TbN), trabecular separation (Tb.Sp), and connectivity density (Conn.D) in the stress and control groups. Values represent mean \pm SD ( $n=8$ per group; ${ }^{*} P<0.05$, ${ }^{*} P<0.01$; Student's $t$ test). (F) Correlation analysis of BV/TV and time spent in central area/open arms $(n=16)$. (C) Immunostaining of SF1 neurons in the VMH. Scale bar: $150 \mu \mathrm{m}$. (H) Immunostaining of vesicular glutamate transporter (Vglut2) in the VMHdm. Scale bar: $75 \mu \mathrm{m}$. dm/c, dorsomedial and central parts of the VMH; vl, ventrolateral part of the VMH (I) Immunostaining of GAD65-positive GABAergic projections in the VMHdm. Scale bar: $75 \mu \mathrm{m}$. (J) Quantification of GABA levels in the VMHdm region in stress and control groups. Values represent mean \pm SD ( $n=6$ per group; ${ }^{* *} P<0.01$; Student's $t$ test). (K) Representative inhibitory postsynaptic currents (IPSCs) of SF1 neurons in the VMHdm in the stress and control groups. (L) Quantification of the frequency and amplitude of IPSCs from SF1 neurons in the stress and control groups. Values represent mean \pm SD ( $n=6$ per group; ${ }^{* *} P<0.01$; Student's $t$ test). 
In this study we found, firstly, that chronic stress in 3 male crewmembers and 1 female crewmember simulating a long-term space mission resulted in both decreased bone density and elevated anxiety levels under normal gravity. We then used a mouse model of unpredictable chronic mild stress to determine the neural circuitry underlying chronic stress-induced bone loss. Second, by combining electrophysiological, optogenetic, and chemogenetic approaches, we found that GABAergic neural circuity in the VMH is responsive in the stress-induced bone loss. Importantly, the GABAergic input in the dorsomedial $\mathrm{VMH}(\mathrm{VMHdm})$ originates in a specific group of somatostatin neurons in the posterior region of the bed nucleus of the stria terminalis (BNST), which is both indispensable for and able to drive stress-induced bone loss. We show that the nucleus tractus solitarius (NTS) and the peripheral sympathetic system were employed by the BNST-VMH neural circuitry to regulate stress-induced bone loss. Our study thus identifies GABAergic neural circuitry from the forebrain to the hypothalamus that is used in the regulation of stress-induced bone loss, reveals a population of somatostatin neurons in the BNST not previously implicated in bone mass regulation, and provides new insights into how stress disorders can influence bone metabolism at a neural-circuits level.

\section{Results}

Chronic stress for 180 days in crewmembers led to decreased bone density and elevated anxiety levels. To investigate the relationship between chronic stress and bone loss, we first performed a Controlled Ecological Life Support System-integrated (CELSS-integrated) experiment in which 3 male crewmembers and 1 female crewmember spent 180 days in an isolated habitat mimicking a space station under normal gravity. Weight, anxiety level, and biochemical parameters were measured in all participants every 30 days during the mission (Supplemental Figure 1A; supplemental material available online with this article; https://doi.org/10.1172/JCI136105DS1). While cortisol levels and weight were stable for each of the 4 crewmembers during the mission (Supplemental Figure 1, B and C), there was an increase in Self-Rating Anxiety Scale measurement scores over time during the prolonged stay in the confined space for 2 of the 3 male participants, but not for the female participant (Supplemental Figure 1D). There was also a significant increase in epinephrine and norepinephrine (NE) levels during the 180 days in all participants (Supplemental Figure 1, E and F), reflecting the elevated level of reported crewmember anxiety. Bone density analysis revealed a statistically significant decrease in average bone mineral density in the femur, femur neck, and lumbar vertebrae in all participants during the mission (Supplemental Table 1 and Supplemental Figure $1, \mathrm{I}-\mathrm{K})$. In addition, the bone formation markers alkaline phosphatase (ALP) and procollagen I carboxy-terminal propeptide (PICP) continuously decreased in all participants (Supplemental Figure 1, $\mathrm{G}$ and $\mathrm{H}$ ). These data consistently show that the 180 days of chronic stress on 4 crewmembers resulted in elevated anxiety levels and decreased bone density.

GABAergic neural circuitry in the dorsomedial VMH is actively involved in a mouse model of chronic stress-induced bone loss. To dissect the neural mechanism underlying chronic stress-induced bone loss, we exposed mice to chronic mild stressors mimicking the CELSS and thus established a mouse model of chronic stressinduced anxiety (Figure 1A). Before chronic stress was applied, entries to, and time spent in, the central area in an open-field (OF) test were similar between the control and stress groups, as were time spent in the open arms and open-arm entries in an elevated plus maze (EPM) test (Supplemental Figure 2, A-D). After 8 weeks of exposure, we assessed anxiety-related behavior again, and, in the OF test, mice in the stress group exhibited significantly fewer entries to, and spent less time exploring, the central area than the control group (Figure 1B). In the EPM test, mice in the stress group exhibited significantly fewer entries to, and spent less time in, the open arms than the control group (Figure 1C). No statistical differences were observed between the stress and control groups in the total distance traveled in the OF, serum cortisol levels, or body weight (Supplemental Figure 2, E-G). However, NE levels were higher and ALP levels were lower in the stress group than in the control group (Supplemental Figure 2, H and I).

Micro-CT analysis of trabecular bones in the proximal tibia revealed that mice in the stress group had a phenotype with obvious low bone mass (Figure 1D). The trabecular bone volume/tissue volume (BV/TV) ratio was 18\% lower in the stress group than in the control group. The trabecular number $(\mathrm{TbN})$ was $15 \%$ lower in the stress group than in the control group, accompanied by $13 \%$ higher trabecular separation (Tb.Sp) (Figure 1E), illustrating lower bone mass in the stress group. Linear regression analysis using BV/TV and time spent in central area/open arms revealed a strong relationship and inverse association between BV/TV and behavioral parameters reflecting anxiety levels (Figure $1 \mathrm{~F}$ ). In vivo calcein labeling confirmed that rates of new bone formation were lower in the stress group than in the control group (Supplemental Figure 3, A and B). ALP staining showed that ALP expression level was attenuated in the stress group (Supplemental Figure 3C) and that the number of ALP-positive osteoblasts per bone surface area was significantly lower in the stress group than in the control group (Supplemental Figure 3D). Tartrate-resistant acid phosphatase (TRAP) staining and receptor activator of NF- $\mathrm{KB}$ ligand (RANKL) immunostaining were conducted to evaluate bone resorption. We found much stronger TRAP and RANKL staining of trabecular bone in the stress group compared with the control group (Supplemental Figure 3, E and G). Quantification revealed that the numbers of RANKL-positive cells and of TRAP-positive osteoclasts per bone surface area were significantly higher in the stress group (Supplemental Figure 3, F and H). Serum carboxy-terminal collagen cross-links-1 (CTX-1) and RANKL levels were higher in the stress group than in the control group, suggesting increased bone resorption during the chronic stress (Supplemental Figure 3, I and J).

The dorsomedial VMH (VMHdm) is important in regulating anxiety (23), energy balance (13), and bone metabolism (25). The major type of neurons in the VMHdm are SF1 neurons, which are mainly glutamatergic (Figure $1, \mathrm{G}$ and $\mathrm{H}$ ), and thus we next began to dissect the neural circuit facilitating the bone resorption mechanism starting from the VMHdm. Dense GAD65-positive GABAergic projections were observed in the VMHdm (Figure 1I). Microdialysis revealed significantly higher VMHdm GABA levels in the stress group than in the control group (Figure 1J). We then recorded inhibitory postsynaptic currents (IPSCs) in SF1 neurons in the VMHdm and found a significantly elevated frequency of spontaneous IPSCs in the stress group than in the control group (Figure 1, K and L). Both 
A VGAT mouse

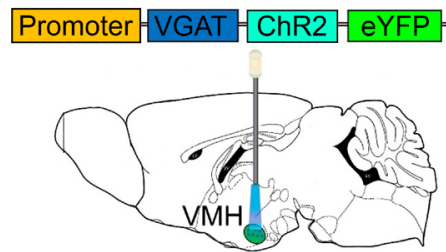

D
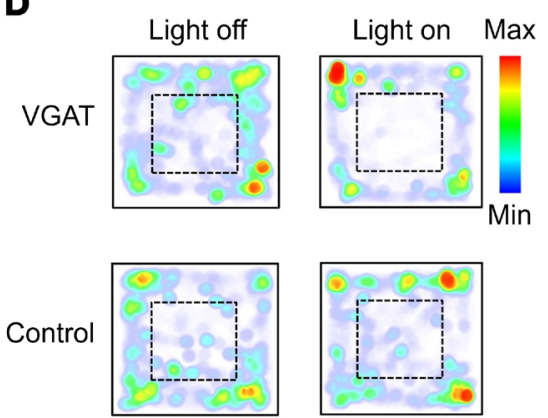

B

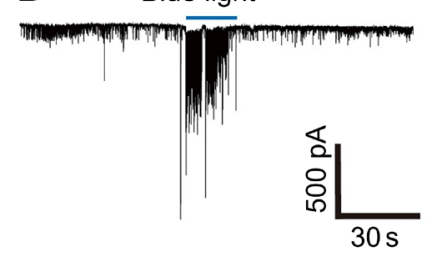

E
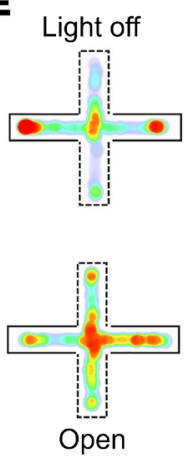

C

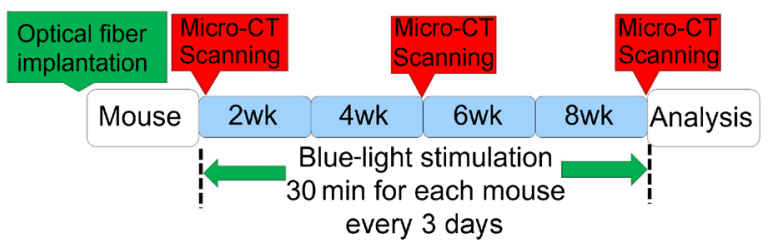

$\mathbf{F}$

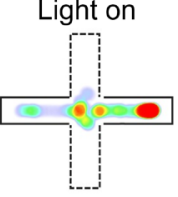

$\mathbf{G}$
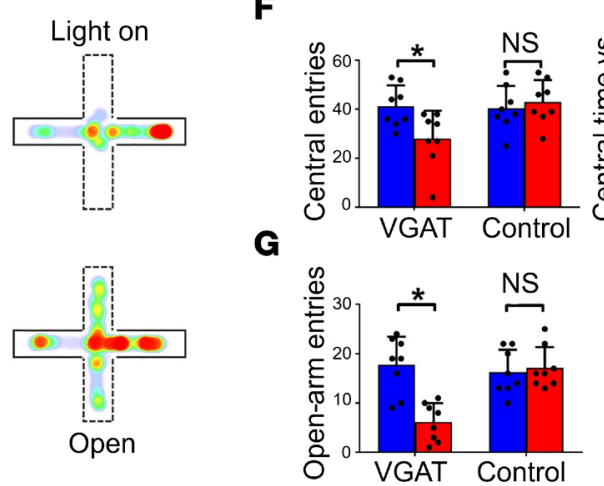

8 weeks
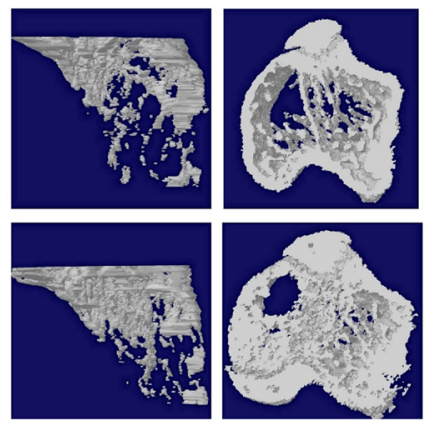
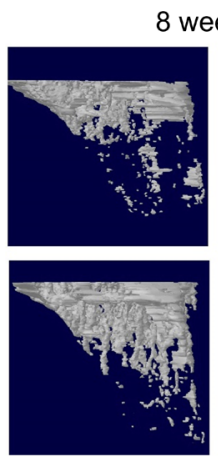
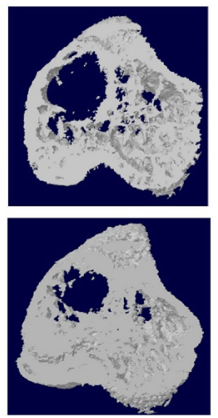
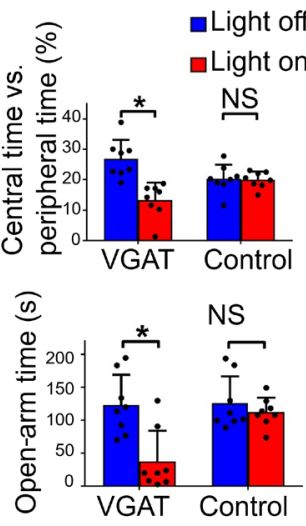

H

Control

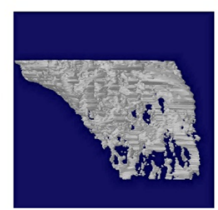

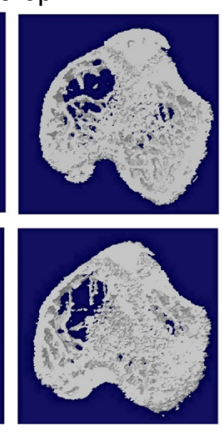
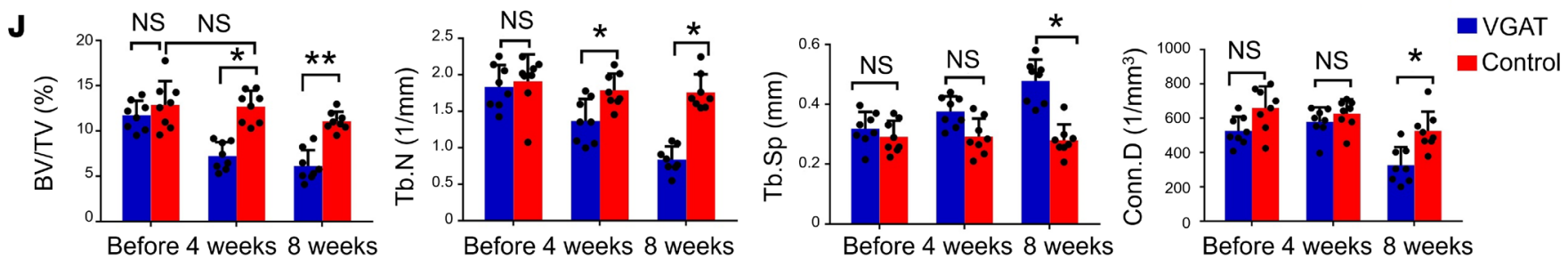

Figure 2. Activation of GABAergic projections in the VMHdm induces anxiety-like behaviors and bone loss. (A) Optical fibers were implanted into the VMHdm of VGAT-ChR2 mice, and the region was illuminated with blue light. (B) Electrophysiological recording of a typical IPSC from SF1 neurons during blue-light stimulation. (C) Schematic showing the schedule of blue-light stimulation and bone analysis with intermittent micro-CT scanning. (D) OF test of VGAT and control mice before and during blue-light stimulation. VGAT mice displayed obvious anxiety-like behavior. (E) EPM test of VGAT and control mice before and during blue-light stimulation. VGAT mice displayed obvious anxiety-like behavior. (F) Quantification of entries to, and time spent in, the central area before and during blue-light stimulation in the VGAT and control groups. Values represent mean \pm SD $\left(n=8\right.$ per group; ${ }^{*} P<0.05 ; 1$-way ANOVA with Bonferroni's correction for comparisons). (G) Quantification of entries to, and time spent in, the open arms in the VGAT and control groups before and during blue-light stimulation. Values represent mean $\pm S D\left(n=8\right.$ per group; ${ }^{*} P<0.05$; 1 -way ANOVA with Bonferroni's correction for multiple comparisons). (H) Micro-CT analysis of VGAT and control group bone structure before, during, and after blue-light stimulation. A significantly low-bone mass phenotype was observed in the VGAT group 4 and 8 weeks after light stimulation began. (I) H\&E staining of the proximal tibia in the VGAT and control groups at 8 weeks after stimulation. Scale bars: $200 \mu \mathrm{m}$. (J) Micro-CT analysis of BV/TV, TbN, Tb.Sp, and Conn.D in the VGAT and control groups. Values represent mean \pm SD ( $n=8$ per group; ${ }^{*} P<0.05,{ }^{* *} P<0.01$; 1-way ANOVA with Bonferroni's correction for multiple comparisons).

frequency and amplitude of IPSCs were completely blocked by $50 \mu \mathrm{M}$ of bicuculline, a competitive antagonist of $\mathrm{GABA}_{\mathrm{A}}$ receptors (Supplemental Figure 2, L-N), suggesting that GABAergic neural projections in the VMHdm play an important role in the mouse model of stress-induced bone loss.
Since the cocaine- and amphetamine-regulated transcript (CART), an important neurotransmitter that plays a key role in the central control of bone remodeling, is involved in the inhibition of bone resorption by modulating RANKL signaling (26), we then investigated CART mRNA levels. We determined CART mRNA 
expression levels in the VMH of stressed and control mice using quantitative PCR (qPCR) and found that expression was $60 \%$ lower in the stress group than in the control group (Supplemental Figure 2J). We also measured CART concentration in the VMH using microdialysis. We found that CART concentration was significantly lower in the stress group than in the control group (Supplemental Figure $2 \mathrm{~K}$ ), suggesting increased bone resorption in the stress group.

Activation of GABAergic projections in the VMHdm inhibits the firing of SF1 neurons and induces anxiety-like behavior and bone loss. To interrogate the function of GABAergic projections in the VMHdm during stress-induced bone loss, we used transgenic mice expressing channelrhodopsin-2 (ChR2) under the promoter of the conditional allele of the vesicular GABA transporter (VGAT) (27), which enables the selective activation of GABAergic neurons or terminals using blue light (Figure 2A). VGAT signals surrounded SF1 neurons, and the distributive pattern of VGAT matched that of GAD65, which stains GABAergic axon terminals (Supplemental Figure 4A). We recorded SF1 neurons and found that excitation of GABAergic terminals induced by blue light resulted in fast IPSCs (Figure 2B). The frequency and amplitude of IPSCs induced by blue light were blocked by $50 \mu \mathrm{M}$ of bicuculline (Supplemental Figure 4, B and C). Patch-clamp recordings combined with single-cell reverse transcriptase PCR (RT-PCR) verified that blue light can indeed inhibit the spontaneous firing of SF1 neurons that express the genes vglut2 and Sf1 but not vgat (Supplemental Figure 4, D and E).

To further investigate the causal role of GABAergic projection activation in the VMHdm during stress-induced bone loss, we assessed anxiety behavior and evaluated bone structure changes following activation of the VMHdm in VGAT-ChR2 mice (Figure $2 \mathrm{C}$ ). After a series of light stimulation over 8 weeks, GABA levels in the VMHdm were significantly higher in the VGAT group than in the control group (Supplemental Figure 4F). Both OF and EPM tests showed that VGAT mice displayed obvious anxiety-like behavior during the light-on phase, whereas the control group did not (Figure 2, D and E). During the OF test, there were significantly fewer entries to, and less time spent in, the central area in the VGAT group than in the control group when the blue light was turned on (Figure $2 \mathrm{~F}$ ). Additionally, the VGAT group had significantly fewer entries to, and less time spent in, the open arms in the EPM test than the control group during the light-on phase (Figure $2 \mathrm{G}$ ). Interestingly, microCT analysis revealed continuous bone loss in VGAT group over the weeks of light stimulation (Figure $2 \mathrm{H}$ ). Before light stimulation there were no significant differences in BV/TV, TbN, Tb.Sp, nor connectivity density (Conn.D) between the VGAT and control groups. However, 4 weeks after the light stimulation began, we observed a phenotype marked by obvious low bone mass in the VGAT group (Figure 2J). The VGAT group had 25\% lower BV/TV than the control group, and $\mathrm{TbN}$ was $18 \%$ lower. Eight weeks after light stimulation began, the VGAT group had 28\% lower BV/TV and 26\% lower TbN than the control group, whereas Tb.Sp was $18 \%$ higher (Figure 2J). Histological analysis at 8 weeks confirmed loss of trabecular bone and a larger medulla cavity of the proximal tibia in VGAT mice (Figure 2I). Taken together, these data demonstrate that activation of GABAergic neural terminals in the VMHdm was able to induce anxiety like-behavior and a phenotype marked by low bone mass in the absence of any additional stressors, supporting a direct causal role of VMHdm GABAergic projection activation in stress-induced bone loss.
Somatostatin neurons send GABAergic projections from the BNST to the VMHdm. To investigate the source of GABAergic input to the $\mathrm{VMHdm}$ region, a retrograde virus was used to trace the neuronal connections of SF1 neurons in the VMH region (Figure 3A). Efficient virus dispersion in the $\mathrm{VMH}$ region was obtained, and retrograde monosynaptic transfer of mCherry allowed the mapping of different brain regions with connections to the VMH (Figure $3 \mathrm{~B})$. We found that the main upstream inputs to the VMH included hypothalamic regions, such as the paraventricular nucleus and the anterior hypothalamic nucleus (Figure 3, B and C). Importantly, we also observed dense signals in both lateral dorsal and posterior regions of the bed nucleus of the stria terminalis (BNST), which play a crucial role in stress-related disorders and adaptive anxiety behaviors (Figure 3B). To confirm that the BNST sends GABAergic inputs to the VMHdm, we injected the AAV-Ef1 $\alpha$-DIO-mCherry virus into the BNST region of GAD-Cre mice and observed GABAergic neural projections in the VMHdm region (Supplemental Figure 5, A and B). Further confirmation of neuronal activation in both the lateral dorsal and posterior regions of the BNST in our established mouse model was obtained following staining with c-fos (an immediate-early protein indicating neuronal activation) (Figure 3D). We found that the anxiety group had a significantly higher c-fos-positive cell count than the control group (Figure 3E), suggesting that the BNST sends GABAergic inputs to the VMHdm to regulate stress-induced bone loss. The BNST is composed of multiple subdivisions and contains heterogeneous neuronal subpopulations that mediate different functions (28). To determine the specific neuronal subpopulations projecting to the VMHdm that mediate stress-induced bone loss, we began investigating the types of GABAergic terminals in the VMHdm region. We observed that strong somatostatin nerve terminal signals made synaptic contact with SF1 neurons in the VMHdm, but other subtypes of GABAergic nerve terminals, such as parvalbumin (PV) and cholecystokinin (CCK) nerve terminals, did not (Figure 3, F and G). To determine the specific source of somatostatin from the BNST, we injected AAV9 virus expressing mCherry into the VMHdm of SOM-Cre mice and found a specific population of somatostatin-positive (SOM-positive) neurons that was positively labeled in the lateral posterior region of the BNST (BSTLP) (Supplemental Figure $5 \mathrm{C}$ ). Combining monosynaptic tracing using rabies virus with in situ hybridization of somatostatin mRNA, we confirmed that the identified SOM neurons in the BSTLP region indeed send projections to innervate SF1 neurons in the VMHdm (Figure 3, $\mathrm{H}$ and $\mathrm{K})$. We also observed dense SOM terminals in the VMHdm region when the AAV9 virus was injected into the BSTLP region (Figure 3, I, J, and L). These data consistently show a specific population of SOM neurons in the BSTLP region that send GABAergic neural projections to innervate VMHdm SF1 neurons.

Activation of SOM neural projections from the BNST to the VMH induces anxiety-like behavior and bone loss. To determine whether activation of specific SOM inputs from the BSTLP was able to cause stress-induced bone loss, we injected AAV-Ef1 $\alpha$-DIO-ChR2mCherry virus into the BSTLP region and used blue light to illuminate the VMHdm region (Figure 4A). GABA levels in the VMHdm were significantly higher in the $S O M-C h R 2$ group than in controls (Figure 4B), and SOM neurons were activated in the SOM-ChR2 group after light stimulation (Figure 4D). Electrophysiological 
A

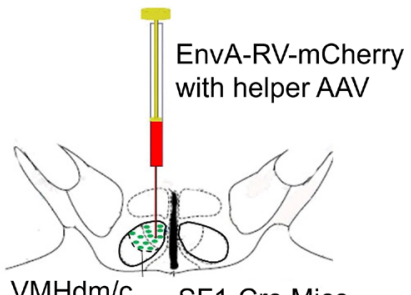

B

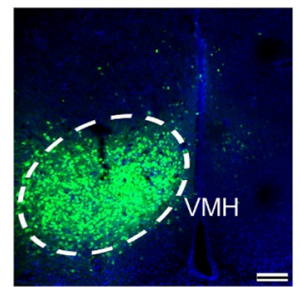

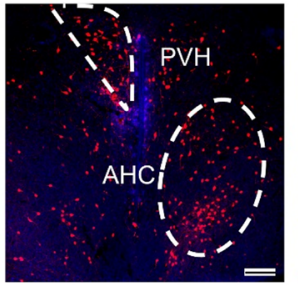

D
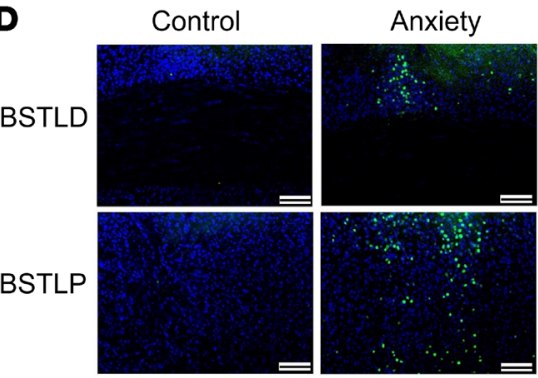

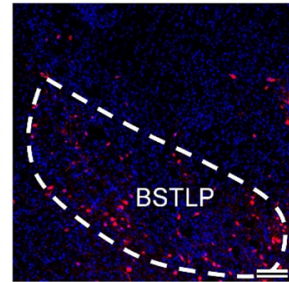

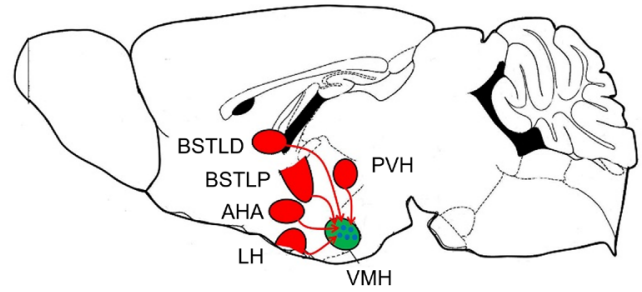

BSTLD

F

PV

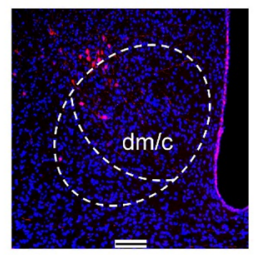

CCK

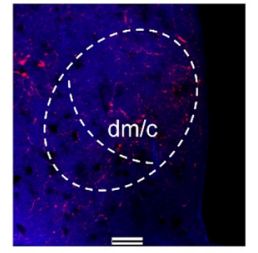

SOM

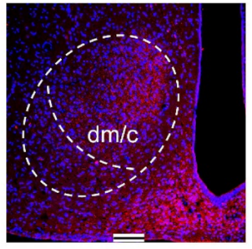

G

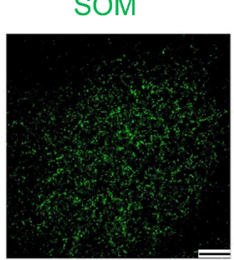

E

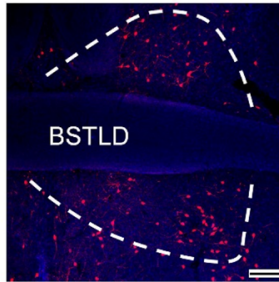

Control $\square$ Anxiety

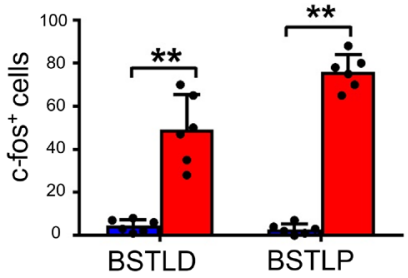

SF1

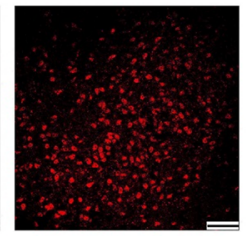

Merge

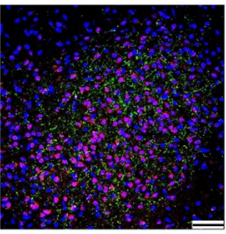

H

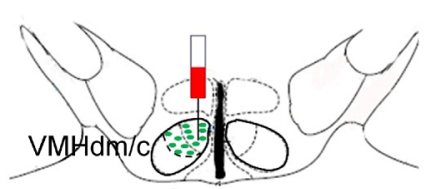

In situ hybridization of somatostatin mRNA in BST
I

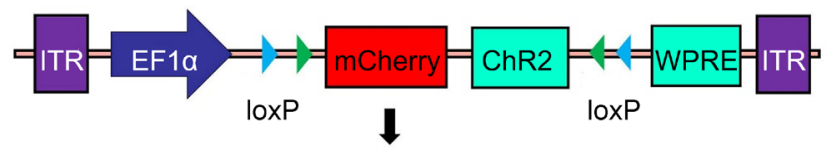

SOM-Cre Mice

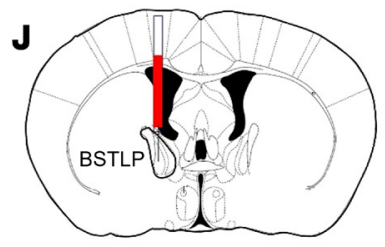

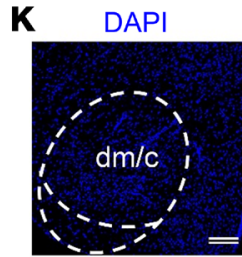

DAPI

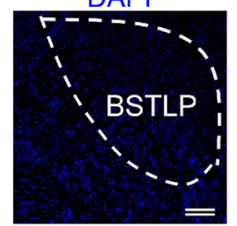

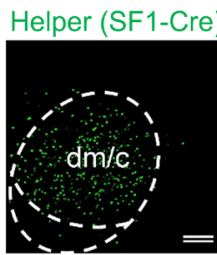

RV

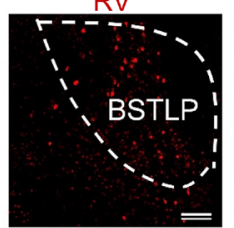

RV

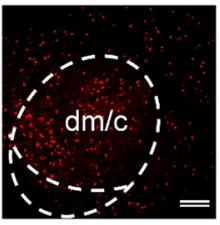

SOM

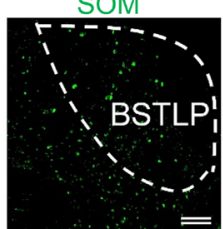

Merge

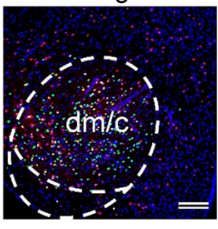

Merge

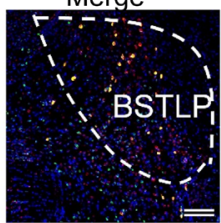

$\mathbf{L}$
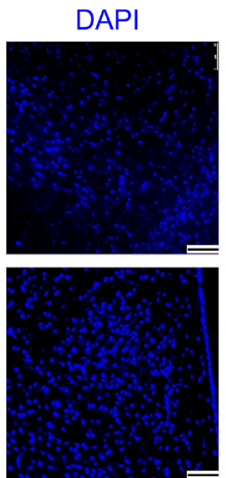

mCherry
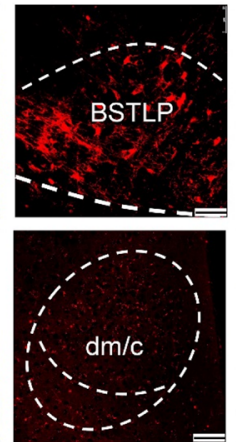

Merge
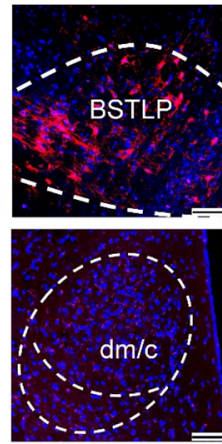

Figure 3. Somatostatin neurons in the posterior BNST send GABAergic projections to the VMHdm region. (A) Schematic showing rabies virus-based (RV-based) monosynaptic retrograde tracing in the SF1-Cre mice. (B) Representative images of the VMH region with the helper AAV virus expressing eYFP, and of RV-labeled cells (red) in different brain regions. PVH, paraventricular nucleus of the hypothalamus; AHC, anterior hypothalamic nucleus; BSTLD, lateral dorsal BNST; BSTLP, lateral posterior BNST. Scale bars: $100 \mu \mathrm{m}$. (C) Schematic mapping of neural circuits from different brain regions to the VMH through monosynaptic neural connection. AHA, anterior hypothalamic area; LH, lateral hypothalamic area. (D) c-fos staining of the BSTLD and BSTLP regions in the anxiety and control groups. Scale bars: $100 \mu \mathrm{m}$. (E) Quantification of c-fos-positive cells in the anxiety and control groups. Values represent mean \pm SD ( $n$ = 3 mice per group; ${ }^{* *} P<0.01$; 1-way ANOVA with Bonferroni's correction for multiple comparisons). (F) Immunostaining of neural projections in VMH. PV, parvalbumin; CCK, cholecystokinin; SOM, somatostatin. Scale bars: $100 \mu \mathrm{m}$. (G) Double staining of SF1 neurons and SOM-positive projections in VMHdm. Scale bars: $50 \mu \mathrm{m}$. (H) RV was injected unilaterally into the dmVMH in SF1-Cre mice, and in situ hybridization of somatostatin mRNA in the posterior region of the BNST (BNSTLP) is shown. (I) Schematic showing AAV9 expressing mCherry and ChR2 under the EF1 $\alpha$ promoter. (J) Schematic showing virus injection into the BNSTLP of SOM-Cre mice. (K) Top: The RV was injected unilaterally into the VMHdm in SF1-Cre mice. Bottom: In situ hybridization of somatostatin mRNA in the BSTLP region. RV signals and somatostatin mRNA were colocalized in the BSTLP region. Scale bars: $100 \mu \mathrm{m}$. (L) Representative image of SOM-positive neurons in the BSTLP (top) and SOM-positive neural projections in the VMHdm (bottom) after injection into the BSTLP region. Scale bars: $100 \mu \mathrm{m}$. 
recordings showed that $20 \mathrm{~Hz}$ pulses of blue light inhibited the firing of SF1 neurons in the VMHdm (Figure 4C). Before light stimulation, entries to, and time spent in, the central area in the OF test were similar between the mCherry-ChR2 and mCherry groups, as were open-arm entries and time spent in the open arms in the EPM test (Supplemental Figure 5, D and E). We then assessed anxiety-related behavior following light stimulation of SOM neural projections. The mCherry-ChR2 group spent less time and made fewer entries into the center during the OF test than the mCherry control group (Figure $4 \mathrm{E}$ ). In the EPM test, the mCherry-ChR2 group also made fewer entries to, and spent less time in, the open arms than the control group (Figure $4 \mathrm{~F}$ ). Thus, the mCherry-ChR2 group displayed higher anxiety-like behavior following light stimulation. Four weeks after light stimulation, bone structure was evaluated, and mice in the mCherry-ChR2 group had a phenotype with a significantly lower bone mass than the mCherry control group (Figure 4G): 20\% lower BV/TV, 25\% lower TbN, and 20\% higher Tb.Sp (Figure $4 \mathrm{H}$ ). We found no statistical differences between groups in total distance traveled in the OF test or in body weight (Supplemental Figure 5, F and G). To confirm the specific function of SOM neural terminals, we optogenetically activated PV-positive neural terminals and CCK-positive terminals in the VMHdm region in $\mathrm{PV}$-Cre mice and CCK-Cre mice, respectively (Supplemental Figure 6, A and B; and Supplemental Figure 7, A and B). In both $\mathrm{PV}$-Cre and CCK-Cre mice, there was no difference in time spent in the central area or entries to the central area in the OF test following light stimulation, in comparison with their respective control groups (Supplemental Figure 6, C and D; and Supplemental Figure 7, C and D). Additionally, micro-CT analysis of bone revealed no changes in BV/TV, TbN, or Tb.Sp (Supplemental Figure 6, E and $\mathrm{F}$; and Supplemental Figure 7, E and F). Together, our data indicate that activation of the specific SOM inputs from the BNST to the VMHdm was able to induce anxiety-like behavior and bone loss, implicating the specificity of the population of SOM neurons in regulating stress-induced bone loss.

Inhibiting the activity of SOM neurons arrests stress-induced bone loss. To further determine the indispensability of this population of SOM neurons in stress-induced bone loss, we first observed enhanced c-fos signals in localized SOM neurons in the stress group (Figure 5B), and that, after staining with anti-corticotrophin-releasing factor (anti-CRF) antibody, some SOM neurons were stained (Figure $5 \mathrm{C}$ ). We then established a stress-induced bone loss model with SOM-Cre mice, and selectively silenced the SOM neurons using the DREADD (Designer Receptor Exclusively Activated by Designer Drugs) technique (Figure 5A). Before chronic stress, entries to, and time spent in, the central area in the OF test were similar between the mCherry and mCherry-hM4Di groups, as were the number of open-arm entries and time spent in the open arms in the EPM test (Supplemental Figure 8, C and D). After this, AAV-Ef1 $\alpha$-DIO-hM4Di-mCherry and AAV-Ef1 $\alpha$-DIOmCherry viruses were injected into SOM-Cre mice, and clozapine $\mathrm{N}$-oxide (CNO) was administered later during testing (Figure 5D). Electrophysiological recordings showed that somatostatin neuronal activity was selectively inhibited by CNO (Supplemental Figure 8, A and B). Anxiety levels were assessed at 8 weeks in OF tests; mice in the mCherry-hM4Di group had more entries to the central area and spent more time in the central area than the
mCherry group (Figure 5E). Similarly, in EPM tests, the mCherry-hM4Di group had significantly more entries to, and spent more time in, the open arms than the mCherry group (Figure $5 \mathrm{~F}$ ). Importantly, bone mass and structure were significantly better in the mCherry-hM4Di group than in the mCherry group (Figure 5G): $25 \%$ higher BV/TV, 20\% higher TbN, and 15\% lower Tb.Sp (Figure $5 \mathrm{H}$ ). We also evaluated baseline bone mass of nonstressed mice in the mCherry and mCherry-hM4Di groups using micro-CT scans, and found that 4 weeks of CNO treatment to inhibit SOM neurons in the BNST did not affect bone mass significantly (Supplemental Figure 8, $\mathrm{H}$ and $\mathrm{I}$ ).

We investigated bone formation and resorption changes after inhibiting SOM neuron activity. In vivo calcein labeling confirmed that rates of new bone formation were higher in the mCherry-hM4Di group than in the mCherry group (Supplemental Figure 9, A and B). ALP staining showed that both ALP expression levels and the number of ALP-positive osteoblasts were significantly higher in the mCherry-hM4Di group than in the mCherry group (Supplemental Figure 9, C and D). Following TRAP staining and RANKL immunostaining, we found much lower TRAP and RANKL expression in the trabecular bone in the mCherry-hM4Di group than in the mCherry group (Supplemental Figure 9, E and G). Quantification revealed that the numbers of RANKL-positive cells and of TRAP-positive osteoclasts were significantly lower in the mCherry-hM4Di group than in the mCherry group (Supplemental Figure 9, F and H). Serum CTX-1 levels and RANKL levels were lower in the mCherry-hM4Di group than in the mCherry group (Supplemental Figure 9, J and K), suggesting decreased bone resorption following inhibition of SOM neuronal activity in stressed mice.

We also investigated CART mRNA levels using $\mathrm{qPCR}$ and found that CART mRNA expression was $50 \%$ higher in the mCherry-hM4Di group than in the mCherry group (Supplemental Figure $8 \mathrm{E})$. We also measured CART concentration in the VMH using microdialysis and found that CART concentration was significantly higher in the mCherry-hM4Di group than in the mCherry group (Supplemental Figure 8F). Together, the DREADD experiments demonstrate that the specific population of SOM neurons in the BSTLP region were indispensable for driving anxiety-like behaviors and induced bone loss, and inhibition of the SOM neuron population led to the arrest of stress-induced bone loss.

Since the raphe-VMH circuit mediates bone accrual through raphe-synthesized serotonin and Htr2c receptors in VMH neurons (15), we further investigated whether the raphe-VMH pathway plays a role in regulating stress-induced bone loss. We established a stress-induced bone loss model with SERT-Cre mice, and injected AAV-Ef1 $\alpha$-DIO-ChR2-mCherry virus into the dorsal raphe nucleus and observed serotoninergic neural projections in the VMHdm region (Supplemental Figure 10, A and B). Next, we used blue light to illuminate the VMHdm region (Supplemental Figure 10A). Before light stimulation, entries to, and time spent in, the central area were similar in the OF test between the mCherryChR2 and mCherry groups, as were open-arm entries and time spent in the open arms in the EPM test (Supplemental Figure 10, $\mathrm{C}$ and $\mathrm{D}$ ). Following the light stimulation, entries to, and time spent in, the central area in the OF test were similar between the mCherry-ChR2 and mCherry groups (Supplemental Figure 10E). The number of open-arm entries and time spent in the open arms 


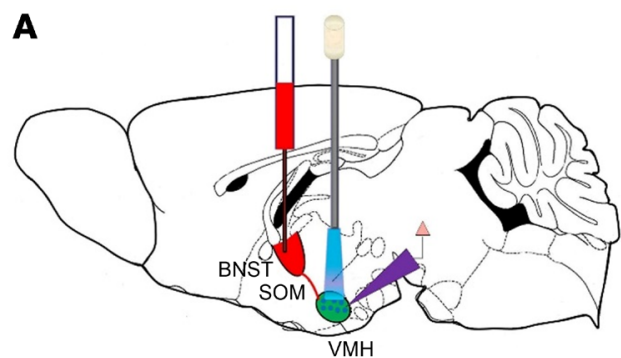

C

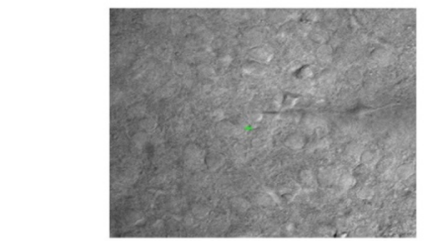

Blue light $20 \mathrm{~Hz} 5 \mathrm{~ms}$

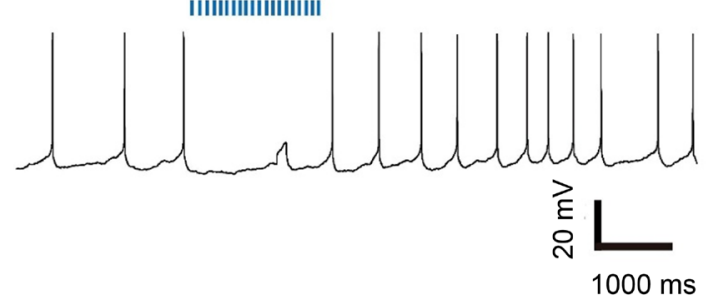

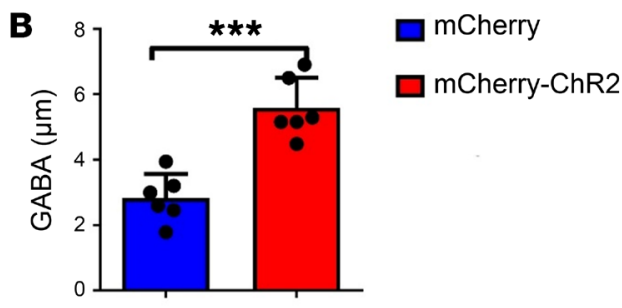

D
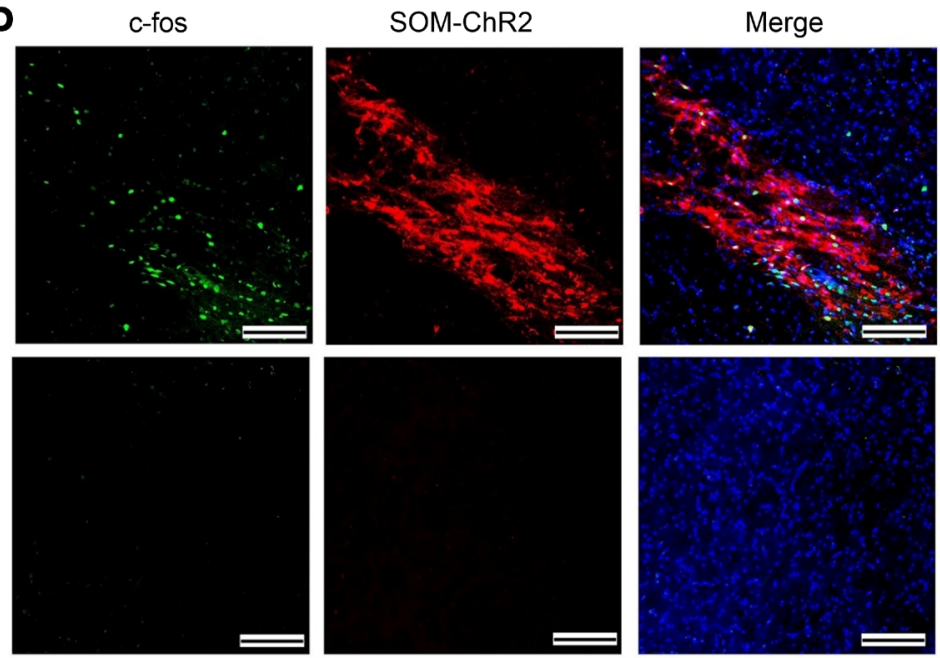

E

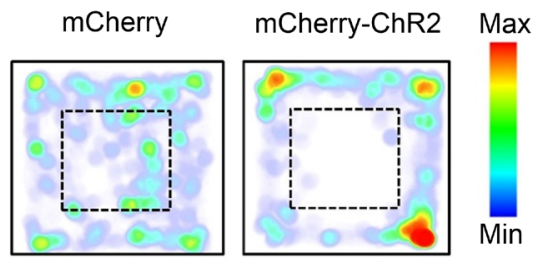

a mCherry a mCherry-ChR2
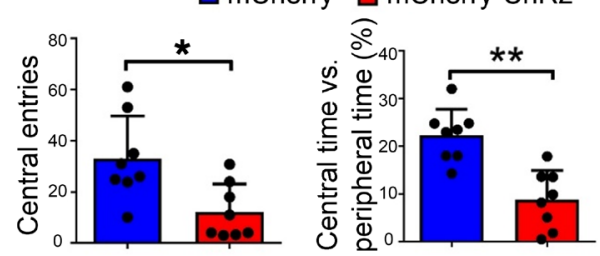

G

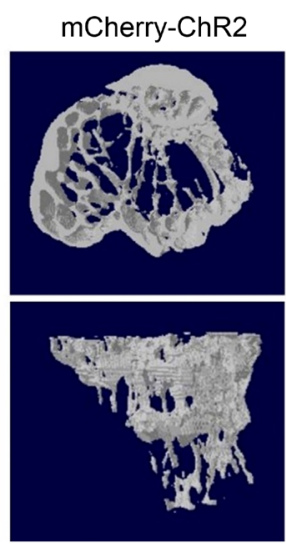

$F$
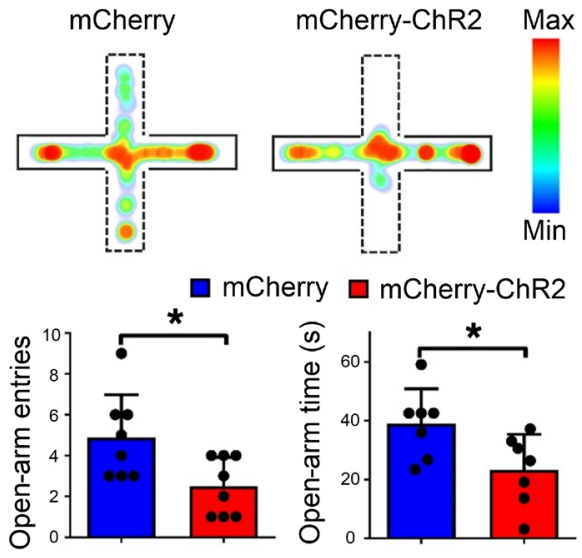

H

aCherry $\square$ mCherry-ChR2
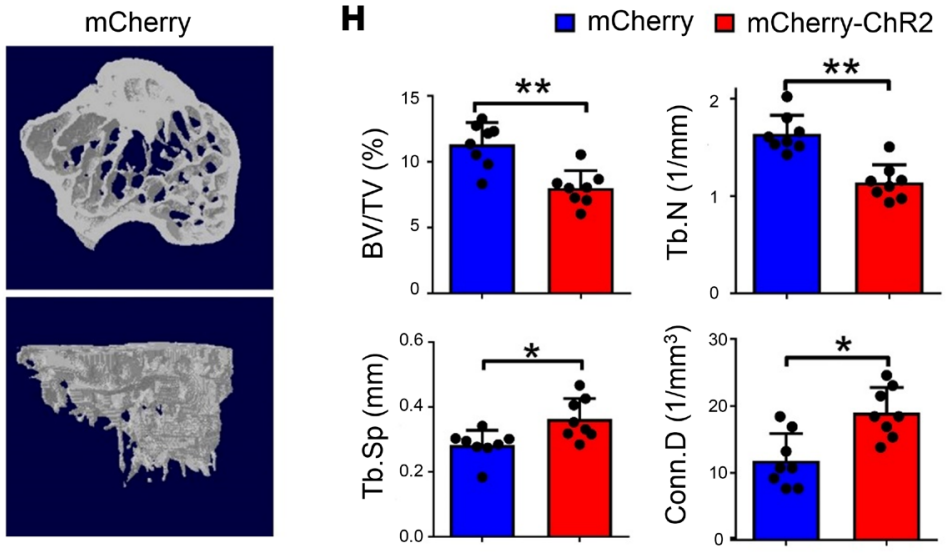
Figure 4. Activation of the SOM neural projection from the BNST to the VMH triggers bone loss in the absence of stressors. (A) Schematic showing AAV-Ef1 $\alpha$-DIO-ChR2-mCherry virus injected into the BSTLP region. Blue light was used to illuminate the $\mathrm{VMHdm}$ region containing SOM neural terminals. (B) Quantification of the GABA level in SOM-ChR2 and SOM-eYFP groups. Values represent mean \pm SD ( $n=6$ per group; ${ }^{* *} P$ $<0.001$; Student's $t$ test). (C) Representative electrophysiological recordings showing inhibition of SF1 neuron firing following stimulation of the VMHdm with light in SOM-ChR2 mice. (D) c-fos staining of SOM-positive neurons in mCherry-ChR2 group (top) and control (bottom) after bluelight stimulation. Scale bars: $100 \mu \mathrm{m}$. (E) OF test comparing mCherryChR2 and mCherry groups. Mice in the mCherry-ChR2 group made significantly fewer entries to the central area and spent less time exploring the central area. Values represent mean \pm SD ( $n=8$ per group; ${ }^{*} P<0.05$, ${ }^{*} P<0.01$; Student's $t$ test). (F) EPM test comparing mCherry-ChR2 and mCherry groups. mCherry-ChR2 mice exhibited significantly fewer entries and spent less time in the open arms. Values represent mean \pm SD $(n=8$ per group; ${ }^{*} P<0.05$; Student's $t$ test). (G) Micro-CT analysis of the bone structure of mCherry-ChR2 and mCherry groups 4 weeks after light stimulation began. A significantly low-bone mass phenotype was observed in the mCherry-ChR2 group compared with the mCherry group. (H) Micro-CT analysis showing BV/TV, TbN, Tb.Sp, and Conn.D in mCherry-ChR2 and mCherry groups. Values represent mean $\pm \mathrm{SD}\left(n=8\right.$ per group; ${ }^{*} P<0.05$, ${ }^{* *} P<0.01$; Student's $t$ test)

in the EPM test were also similar between the mCherry-ChR2 and mCherry groups (Supplemental Figure 10F). Four weeks after blue-light stimulation, bone structure was evaluated, and mice in the mCherry-ChR2 group had a phenotype with significantly higher bone mass than the mCherry control group (Supplemental Figure $10 \mathrm{G}$ ): $\mathrm{BV} / \mathrm{TV}$ ratio was $50 \%$ higher and $\mathrm{TbN}$ was $25 \%$ higher in the mCherry-ChR2 group than in the mCherry group, accompanied by $13 \%$ lower $\mathrm{Tb} . \mathrm{Sp}$ in the mCherry-ChR2 group than in the mCherry group (Supplemental Figure 10H). Collectively, these data suggest that the raphe-VMH circuit plays a crucial role in arresting stress-induced bone loss, but is not involved in stress-induced anxiety-like behavior.

NTS is downstream of the VMHdm and necessary for bone loss induced by SOM neurons. Stimulation of SOM neural projections from the BNST to the VMHdm resulted in bone loss in the absence of stressors, and SOM neurons were indispensable for stress-induced bone loss. We were next interested in determining the downstream effectors of this neural circuit. SF1 neurons send neural projections to regulate autonomous activities (29), and we injected a retrograde virus into the marrow compartment of the trabecular tibia bone in mice to retrogradely label the neural circuitry innervating the bone (Supplemental Figure 11A). In addition to the VMH and BNST, we observed strong signals in the nucleus tractus solitarius (NTS), which is known to modulate sympathetic activity $(30,31)$ (Supplemental Figure 11B). Next, we injected AAV-Efl $\alpha$-DIO-mCherry virus precisely into the VMHdm region of SF1-Cre mice (Figure 6A) and observed, interestingly, that SF1 neurons did indeed send neural projections to the medial solitary nucleus (SolM; Figure 6B), which is a subregion of NTS that contains predominantly Vglut2 neurons (Figure 6C). We then confirmed the BNST-VMH-NTS neural pathway using a TRIO experiment (viral-genetic tracing of the input-output organization of the central neural circuit) (Figure 6D), and SF1 neurons that were innervated by upstream BNST neurons did indeed send direct neural projections to the NTS region (Figure 6E). To further confirm the function of localized downstream Vglut 2 neurons in bone loss, we selectively inhibited those Vglut2 neurons in the NTS specifically innervated by SF1 neurons using the DREADD technique (Figure 6F). We found that the hM4Di gene was selectively expressed in SolM (Supplemental Figure 11D), and inhibition of Vglut2 neurons resulted in higher NE levels in the hM4Di group than in the eYFP group (Supplemental Figure 11E). We analyzed bone structure following chemogenetic inhibition of Vglut2 neurons for 4 weeks using micro-CT and found that, compared with the eYFP group, the hM4Di group had significantly lower bone mass and bone structure (Figure 6G): 15\% lower BV/TV, 18\% lower TbN, and 10\% higher Tb.Sp (Figure 6H). These data suggested that the Vglut2 neurons in SolM were specifically employed by SF1 neurons to regulate stress-induced bone loss.

Immunostaining showed that tyrosine hydroxylase (TH), a marker for sympathetic nerve axons, could be identified in both tibia bone and bone marrow (Figure 6I). We cultured osteoprogenitor cells from murine bone marrow and found that $\beta_{2}$ - and $\beta_{3}$-adrenergic receptors were positively expressed in osteoprogenitor cells (Supplemental Figure 11F). We had also found that the SOM-ChR2 group and the stress group had higher NE levels than the control group (Supplemental Figure 8G), so we then further investigated the effects of NE in the serum on the osteogenic differentiation process. We quantified ALP and found that osteoblastic differentiation of osteoprogenitors was significantly inhibited by serum from the stress group compared with that of the control group (Supplemental Figure 11G). Using RT-PCR analysis, we found that the normalized expression of Runx2, Alp, Col1a1, and Opn was significantly lower in the osteoprogenitors treated with the serum from the stress group than in those treated with the serum from the control group at 2 weeks of osteogenic differentiation (Supplemental Figure 11H). The inhibitory effects of sympathetic regulation on osteogenesis in vivo were also confirmed using gene expression analysis with freshly isolated bone marrow cells, and we found that the expression of Runx2, Alp, Col1a1, and Opn was lower in the stress group than in the control group. However, treatment using beta blockers significantly elevated the expression of these osteogenesis-related genes (Supplemental Figure 11I).

To confirm the sympathetic regulation of bone mass, we used the DREADD technique to selectively silence TH-positive nerve fibers in bone marrow of $\mathrm{TH}$-Cre mice (Figure 6J). The hM4Di group had significantly higher bone mass compared with the TH-Cre control group: 35\% higher BV/TV and 29\% higher TbN (Figure 6K). To further investigate whether $\beta_{2}$-adrenergic receptors in osteoblasts were involved in the sympathetic regulation of bone mass, we bred $A d r b 2_{O C}{ }^{-1-}$ mice, and then exposed them along with control mice to the chronic mild stressors (Supplemental Figure 12, A and B). Before chronic stress, entries to, and time spent in, the central area in the OF test were similar between control and $A d r b 2_{O C}{ }^{-1-}$ groups, as were the number of open-arm entries and time spent in the open arms in the EPM test (Supplemental Figure 12 , C and D). Following chronic stress, there was no difference in time spent in the central area or entries to the central area in the OF test between the control and Adrb2 ${ }_{o c}^{-/-}$groups (Supplemental Figure 12E). Open-arm entries and time spent in the open arms in the EPM test were also similar between the control and $A d r b 2_{O C}{ }^{-1-}$ 
A

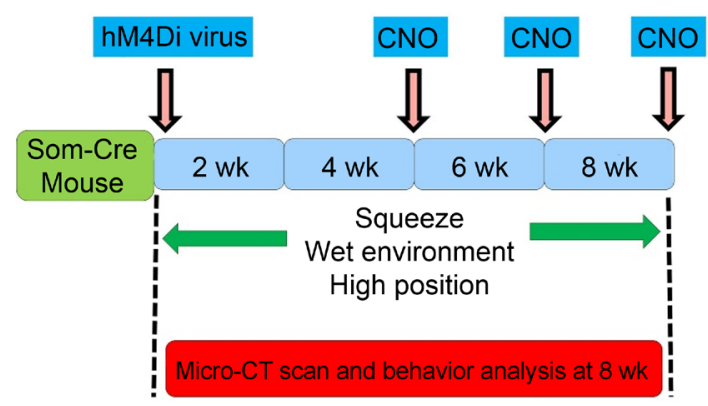

B
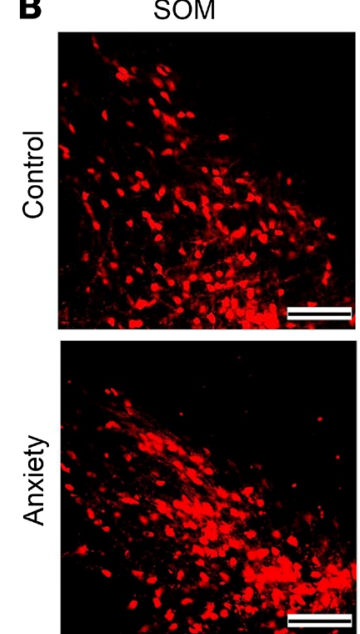

c-fos
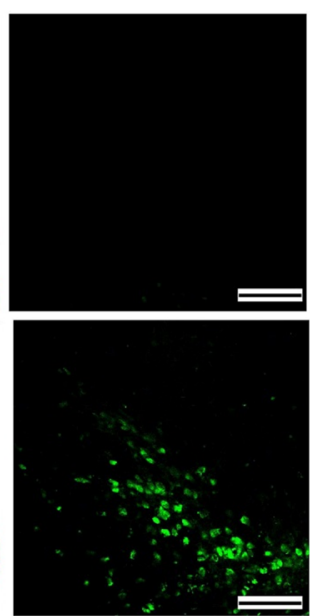

Merge

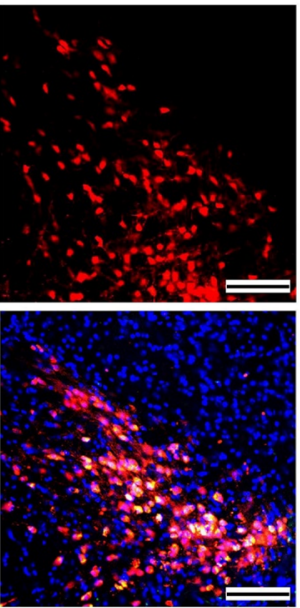

C

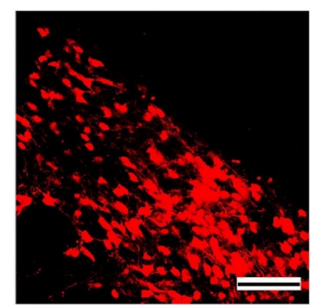

$\mathbf{E}$
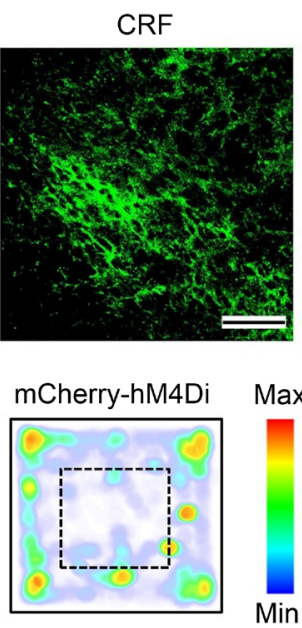

M

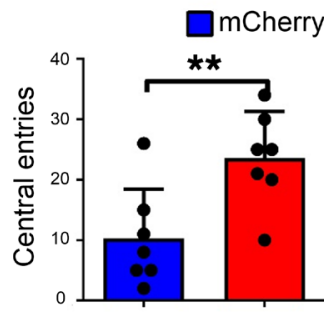

$\square$ mCherry-hM4Di

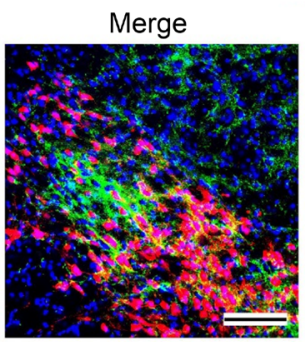

D

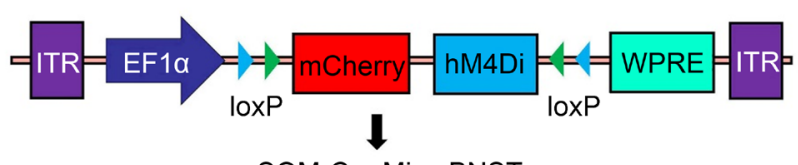

SOM-Cre Mice BNST

$\mathbf{F}$

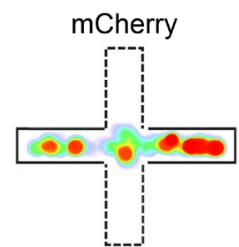

mCherry-hM4Di

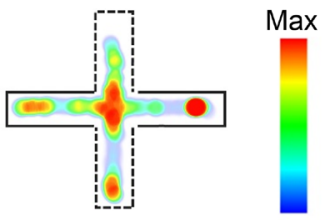

$\square$ mCherry $\square$ mCherry-hM4Di Min

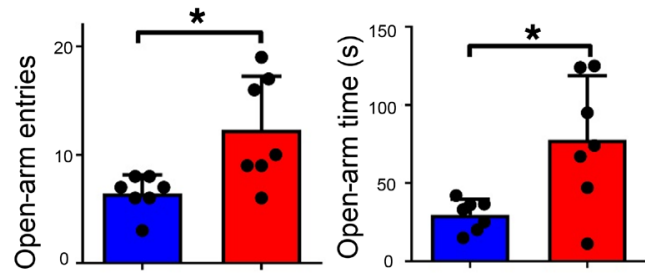

G
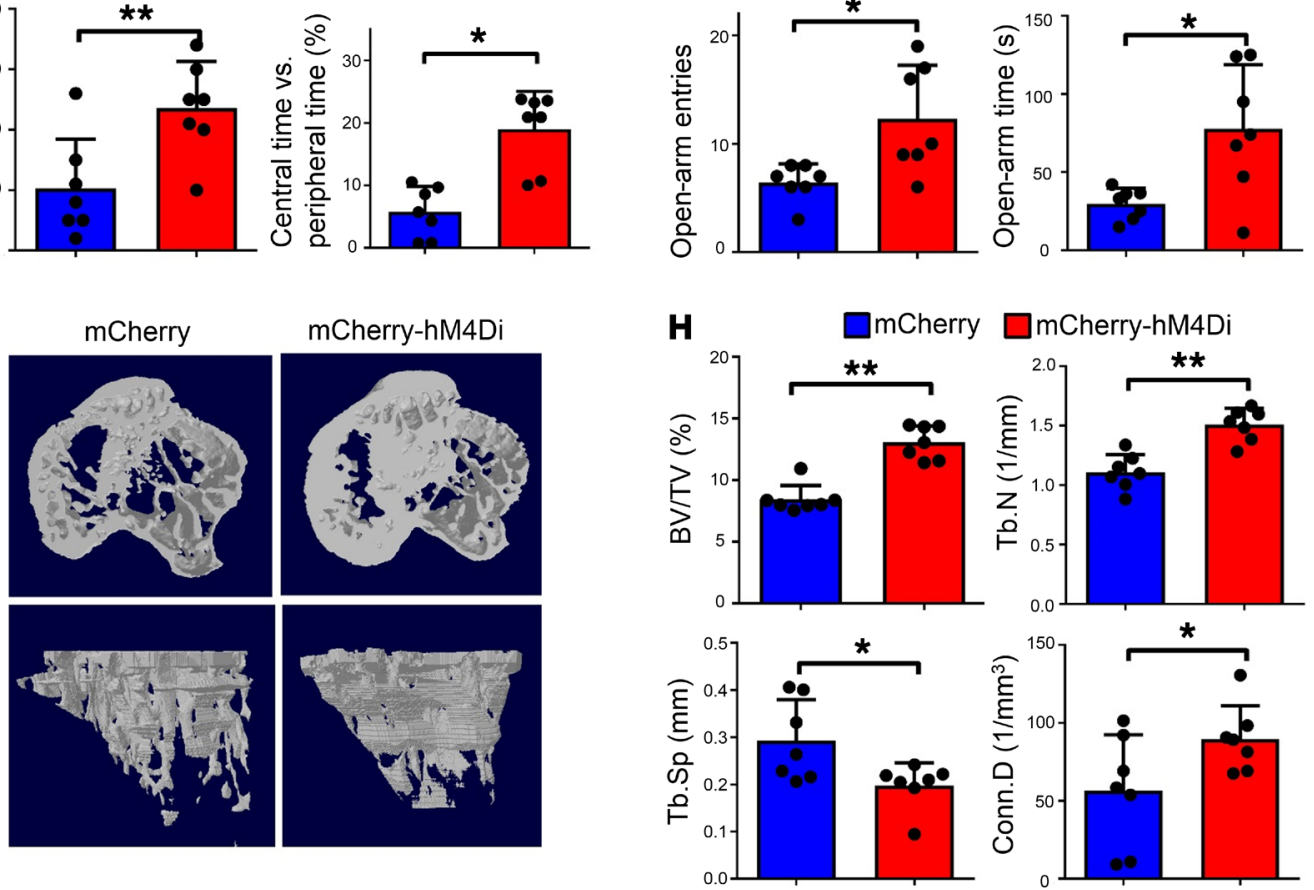
Figure 5. Somatostatin neurons in the BNST are indispensable for stress-induced bone loss. (A) Schematic showing SOM neuron silencing using the DREADD (Designer Receptor Exclusively Activated by Designer Drugs) technique in the stress-induced bone loss model. Clozapine $\mathrm{N}$-oxide (CNO) was used at 4, 6, and 8 weeks. (B) c-fos staining of somatostatin-positive cells in anxiety and control groups. Enhanced positive signals were observed in the anxiety group. Scale bars: $50 \mu \mathrm{m}$. (C) Immunostaining of corticotrophin-releasing factor (CRF) on somatostatin-positive neurons in the stress-induced bone loss model with SOM-Cre mice. Scale bars: 50 $\mu \mathrm{m}$. (D) Schematic showing AAV-Ef1 $\alpha$-DIO-hM4Di-mCherry virus injection into the BNST region of SOM-Cre mice. (E) OF test comparing mCherry and mCherry-hM4Di groups. Mice in the mCherry-hM4Di group made significantly more entries to the central area and spent more time exploring the central area. Values represent mean \pm SD $\left(n=7\right.$ per group; ${ }^{*} P<0.05$, ${ }^{*} P<$ 0.01 ; Student's $t$ test). (F) EPM test comparing mCherry and mCherry-hM4Di groups. The mCherry-hM4Di group exhibited significantly more entries to the open arms and spent more time there. Values represent mean \pm SD ( $n=7$ per group; ${ }^{*} P<0.05$; Student's $t$ test). (C) Micro-CT analysis of bone structure comparing mCherry and mCherry-hM4Di groups after inhibition of SOM neurons. The reduced-bone mass phenotype was arrested in the mCherry-hM4Di group. (H) Micro-CT analysis of BV/TV, TbN, Tb.Sp, and Conn.D in the mCherry and mCherry-hM4Di groups. Values represent mean $\pm \mathrm{SD}\left(n=7\right.$ per group; ${ }^{*} P<0.05,{ }^{*} P<0.01$; Student's $t$ test $)$.

groups (Supplemental Figure 12F), but were both lower than prestress levels. Eight weeks after chronic stress, bone structure was evaluated, and mice in the $A d r b 2_{O C}{ }^{-/-}$group had a phenotype with a larger bone mass than the control group (Supplemental Figure $12 \mathrm{G}): \mathrm{BV} / \mathrm{TV}$ ratio was $25 \%$ higher and $\mathrm{TbN} 20 \%$ higher in the stress group than in the control group, accompanied by $10 \%$ lower Tb.Sp in the $A d r b 2_{O C}{ }^{-1-}$ than in the control group (Supplemental Figure $12 \mathrm{H}$ ), suggesting that osteoblastic expression of $\beta_{2}$-adrenergic receptors was necessary for the stress-induced bone loss. Collectively, these data consistently demonstrate that $\mathrm{NE}$ and $\beta_{2}$-adrenergic receptors in the peripheral sympathetic system relay the BNSTVMH-NTS neural pathway to modulate stress-induced bone loss.

\section{Discussion}

The brain plays an essential role in the integration of cognitive and emotional neural information to regulate food intake and to maintain peripheral metabolic homeostasis. A close association between bone metabolism disturbances and psychiatric disorders, such as anxiety, has been recently reported (1-3); however, the neural mechanism behind this was unclear. To our knowledge, our study is the first to provide experimental evidence that the BNST-VMH-NTS neural circuitry can regulate stress-induced bone loss. We identified a specific group of somatostatin neurons in the posterior region of the BNST projecting to the VMH that is responsible for stress-induced bone loss. These results not only indicate that the GABAergic neural circuitry from the forebrain to the hypothalamus can exert a fundamental influence on the homeostasis of bone metabolism, but they also provide a new central target for therapeutic intervention in stress-induced bone metabolism disorders.

Stress-related disorders, central molecules, and neural circuitry regulating bone metabolism. Recent studies have suggested that mood disorders and neurodevelopmental disorders are closely related to bone metabolism abnormalities. For example, bone quality is lower among men and women with a history of mood disorders (5), and bone development is compromised in children with the autism spectrum disorders (32). Psychological changes due to extreme environments, such as prolonged simulated spaceflight, can also lead to bone metabolism disorders (9-11). While microgravity is an established cause of bone loss during spaceflight, bone formation in astronauts progressively deteriorates under normal gravity after returning to earth (9). In this study, we performed a longitudinal study of the effects of chronic stress on anxiety and bone loss in crewmembers under normal gravity. We observed significantly raised anxiety scores and continuous elevated levels of NE during the period of chronic stress. Lower bone mineral density and a continuous low level of bone formation markers also reflected impaired bone formation during the experiment. Although there were only 4 participants, our longitudinal data consistently demonstrate that chronic stress in crewmembers indeed resulted in both decreased bone density and elevated anxiety levels under normal gravity.

Previous clinical studies have also demonstrated that high levels of anxiety in older men but not women may increase their risk of fracture (33). Older men with probable anxiety have a more than 4-fold risk of experiencing a fracture (4). Heikkinen et al. also found that mood and anxiety disorders are associated with lower bone mineral density in men (34). In our study, the Self-Rating Anxiety Scale measurements showed increased scores during the prolonged stay for 2 of the 3 male participants, but not for the female participant, which implied that sex might be a factor influencing anxiety levels after the stress.

Central molecules and neuropeptides can play important roles in the regulation of bone metabolism (35). Leptin inhibits bone formation through the sympathetic nervous system (14), and orexin is a critical regulator of skeletal homeostasis and exerts dual regulation of bone mass (16). Specific neuronal subtypes and circuits can also exert influence on the regulation of bone mass (36), such as AGRP neurons in the arcuate nucleus that regulate bone mass through the sympathetic nervous system (37). While genetic knockdown of CamKK $\beta$ and CamKIV in SF1 neurons in the VMH leads to severe low bone mass (21), serotonin neurons in the raphe nucleus send axonal projections to VMH SF1 neurons and promote bone mass through Htr2c receptors on SF1 neurons (15). These genetic and molecular studies provide useful information regarding neural molecules that regulate bone mass. However, until now, studies attempting to dissect the central neural regulation of bone at the circuit level have been scarce and the specific neural circuits regulating stress and bone mass unclear. We used an established mouse model to examine the neural circuitry regulating stress-induced bone loss and identified a neural circuit from the forebrain to the hypothalamus that relayed anxiety information and regulated bone mass. Specifically, GABAergic neural projections in the VMH can regulate anxiety-like behavior and reduce bone mass. Our systematic study using both human participants and animal models thus aids further understanding of the neural mechanism of stress-induced bone loss.

The VMH, an important hypothalamic region, regulates both behavior and bone metabolism. The VMH is heavily involved in regulating energy metabolism and glucose homeostasis (38-40). For example, leptin can directly activate SF1 neurons in the VMH to regulate normal body weight homeostasis (13), the neural circuit 
A

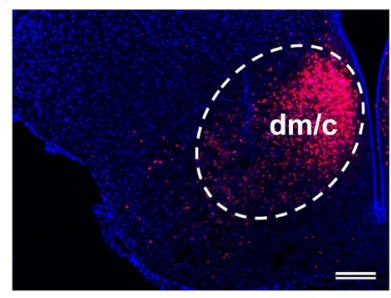

B

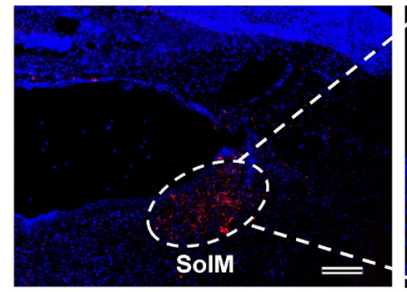

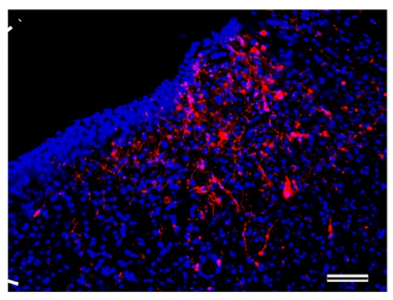

NTS

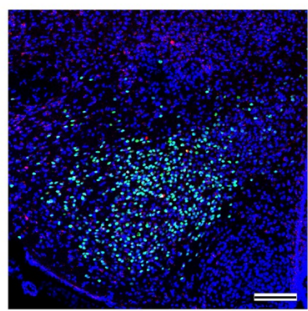

C

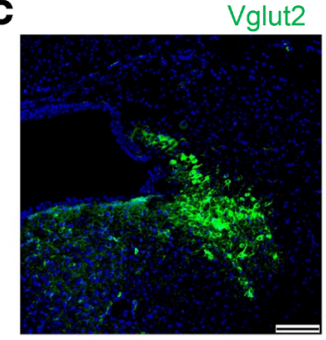

BSTLP
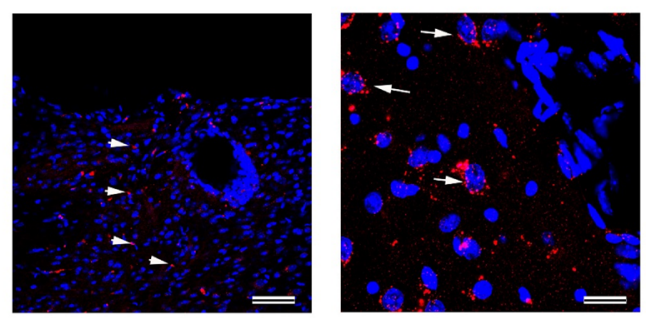

G
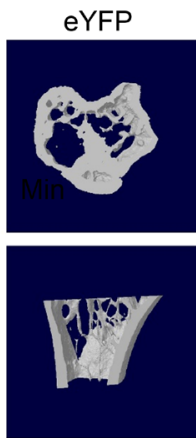

hM4Di
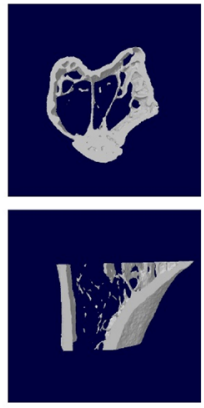

I
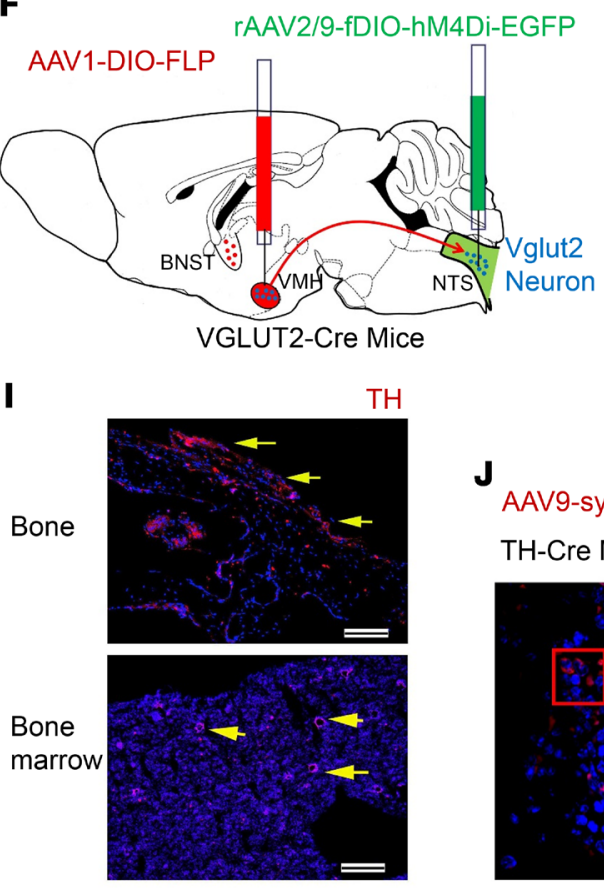

J AAV9-syn-DIO-hM4Di-mCherry TH-Cre Mice

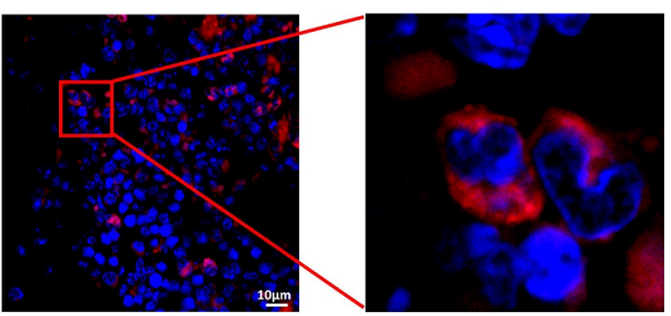

H

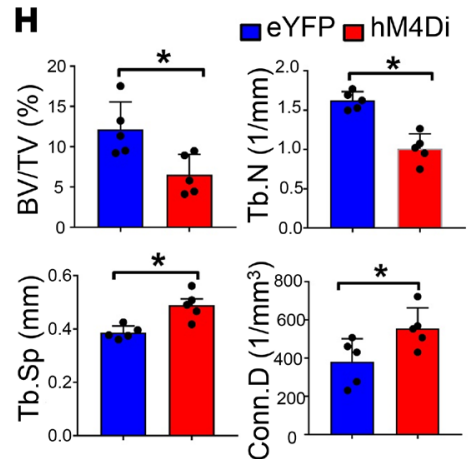

K

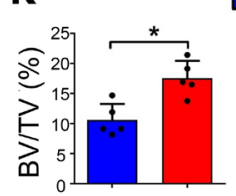

घeYFP $\square \mathrm{hM} 4 \mathrm{Di}$
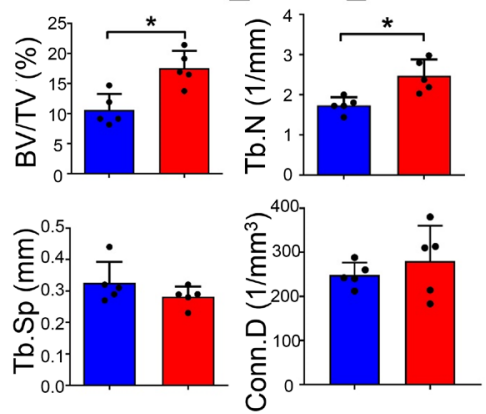

Figure 6. The NTS and the sympathetic system are downstream of SOM neuron-mediated stress-induced bone loss. (A) Representative images of the VMH region with the virus expressing mCherry. Scale bar: $100 \mu \mathrm{m}$. (B) Representative image of neural projections in the SolM, a subregion of the NTS, receiving projections from SF1 neurons in the VMH. Scale bars: $100 \mu \mathrm{m}$ (left), $50 \mu \mathrm{m}$ (right). (C) Immunostaining of Vglut2 in the SolM region of the NTS. Scale bar: $50 \mu \mathrm{m}$. (D) Schematic showing viral genetic tracing of the connection of the BNST-VMH-NTS pathway using the TRIO method. AAV2-DIO-EGFP-TVA+DIO-RVC virus was injected into the VMH region, and RV-EnVA- $\triangle \mathrm{G}$-dsRed virus into the NTS, of SF1-Cre mice. (E) Representative images showing the labeled SF1 neurons in the VMH, SF1-projecting neural terminals in the NTS (arrowheads), and input neurons in the BSTLP region (arrows). (F) Schematic showing silencing of Vglut2 neurons innervated by SF1 neurons using the DREADD technique. AAV1-DIO-FLP virus was injected into the VMH region, and AAV-fDIO-hM4Di-eYFP virus into the NTS, of Vglut2-Cre mice. (C) Micro-CT analysis of bone structure of the eYFP and eYFP-hM4Di groups after inhibition of the Vglut2 neurons. A significant low-bone mass phenotype was observed in the hM4Di group. (H) Micro-CT analysis of BV/TV, TbN, Tb.Sp, and Conn.D in eYFP and hM4Di groups. Values represent mean \pm SD $\left(n=5\right.$ per group; ${ }^{*} P$ $<0.05$; Student's $t$ test). (I) Immunostaining of tyrosine hydroxylase (TH) in bone and bone marrow. Positive signals were observed in both bone and bone marrow (arrows). Scale bars: $100 \mu \mathrm{m}$. () Expression of hM4Di-mCherry on TH-positive nerve fibers in bone marrow of TH-Cre mice. Scale bar: $10 \mu \mathrm{m}$. (K) Micro-CT analysis of BV/TV, TbN, Tb.Sp, and Conn.D in TH-Cre and TH-Cre+hM4Di groups. Values represent mean $\pm S D\left(n=5\right.$ per group; ${ }^{*} P<0.05$; Student's $t$ test).

from the parabrachial nucleus to the VMH controls counterregulatory responses to hypoglycemia $(20,41)$, and serotoninergic projections from the raphe nucleus to the VMH regulate SF1 neurons to promote bone accrual (15). In addition, AP1 alterations in
SF1-expressing neurons in the VMH increase energy, but decrease bone density (24), suggesting that the heterogeneous or distinct neuronal circuits in the VMH mediate the disparate effects of regulating bone homeostasis and energy metabolism. 
The VMH also plays an important role in emotional state, and the dorsomedial part of the VMH (VMHdm) is selectively involved in predator fear (18). Ablation of SF1 neurons in the VMHdm attenuates both innate and learned defensive behaviors (42), and activation of SF1 neurons in the VMHdm can induce defensive-like motor and autonomic responses (23). Serotonin receptors on $\mathrm{VMH}$ neurons contribute not only to the regulation of bone metabolism (15), but also to generalized anxiety (43). In addition to these findings, we show that the link between stress-induced anxiety and bone regulation occurs at the level of the VMHdm. Indeed, our findings show that the activation of the GABAergic neural projections in the VMHdm not only increased anxiety levels, but also led to lower bone mass, thus revealing that GABAergic neural projections in the VMHdm are an important link between anxiety-like behavior and bone metabolism. We believe this study is the first to show that convergent regulation of bone mass and stress is integrated in the GABAergic circuit in the VMHdm; and it provides evidence that psychiatric disorders, such as anxiety, can induce bone loss through GABAergic neural projections in the VMH.

Furthermore, VMH is a dimorphic nucleus that plays a crucial role in sex-based physiological events (44). For instance, some VMH neurons express estrogen receptors, which could be activated by sex hormones, mediating mating, attacking, and social behaviors (45). Frankfurt and colleagues found that the VMH has different neurotransmitter concentrations (GABA and glutamate) during the developmental stage of estrus in females, whereas males show stable GABA and glutamate concentration in this nucleus (46). All these studies suggested the disparate function and regulatory role of the $\mathrm{VMH}$ in male and female mice. Based on this evidence, we chose only male mice to investigate the neuronal mechanism of stress-induced bone loss in the current study.

A GABAergic neural circuit involving the BNST uses somatostatin neurons to regulate stress and bone loss. The BNST is an important brain region in the regulation of stress and anxiety (28). Specific excitatory and inhibitory neuron types in the BNST can use distinct neural pathways to integrate and process aversive stimulation (47). The BNST also serves as a key relay connecting forebrain structures to hypothalamic and brainstem regions that regulate feeding in addition to autonomic and neuroendocrine functions $(48,49)$. The GABAergic inhibitory synaptic input from the BNST to the lateral hypothalamus can suppress local glutamatergic neurons to control food intake (50). In the hypothalamus, both local and long-range GABAergic projections play important roles in regulating behaviors and metabolism (51-53). Our electrophysiological recordings and viral tracing methods show that the activation of GABAergic neural projections from the BNST to the VMHdm can release GABA to inhibit SF1 neurons; this long-range GABAergic axonal projection plays an important role in regulating both anxiety-like behavior and bone loss.

We identified a specific population of SOM neurons in the lateral posterior region of the BNST that sends GABAergic neural projections to the $\mathrm{VMH}$. Importantly, the specific activation of the GABAergic output of these SOM neurons induced both anxiety-like behavior and bone loss in the absence of stressors. Somatostatin-expressing GABAergic neurons constitute a major class of inhibitory neuron in different regions of the brain (54) and are important in anxiety and neuroendocrine function. For example, enhanced activity of SOM neurons in the dorsal BNST drives elevation of anxiety (55), global dysfunction of SOM neurons contributes to altered neuroendocrine function and to aberrant inhibitory neurotransmission (56), and SOM neurons in the hypothalamic arcuate nucleus and tuberal nucleus control energy balance and feeding $(57,58)$. In this study we found that activation of a newly discovered population of SOM neurons in the lateral posterior BNST led to decreased bone mass, and that inhibition of this population of neurons, coupled with anxiety, led to lower anxiety and bone loss than that observed without neural inhibition. These data systematically reveal how SOM neurons in a specific BNST region and its GABAergic output are both indispensable for and able to drive stress-induced bone loss.

The NTS and sympathetic nervous system form a central relay in regulation of bone mass. Periosteum, cortical bone, and bone marrow are all richly innervated by both sympathetic and sensory nerve fibers $(59,60)$. Using virus-based transneuronal tracing from murine bone marrow, we found that neurons in certain brain nuclei, such as the BNST, VMH, and downstream NTS, were retrogradely labeled, thus providing anatomical evidence of central neural control of bone mass. As relays of the CNS, both the sensory and sympathetic nervous systems are crucial in regulating bone homeostasis and metabolism (61-63). For example, sensory nerves in the peripheral cortical femur that express calcitonin gene-related peptide (CGRP) enhance osteogenic differentiation of osteoprogenitor cells (64). The sympathetic nervous system plays important roles in the regulation of bone metabolism (14), and sympathetic tone signals in osteoblasts can inhibit CREB phosphorylation to decrease osteoblast proliferation (61). Our study shows that the sympathetic neurotransmitter norepinephrine (NE) was significantly higher in the anxiety and SOM-ChR2 groups, suggesting elevated sympathetic tone following activation of the BNST-VMH neural projections.

We observed that SF1 neurons send direct axonal projections to a specific region of the NTS, which regulates the activity of the sympathetic nervous system. To further investigate the role of the NTS in relaying the information, we used a TRIO experiment to confirm the BNST-VMH-NTS neural pathway. We then selectively inhibited Vglut2 neurons in the NTS that are specifically innervated by SF1 neurons using the DREADD technique to confirm the function of Vglut2 neurons in the NTS during stress-induced bone loss. Higher levels of NE and lower bone mass and bone structure in the hM4Di group suggest that Vglut2 neurons in the NTS were indeed employed by SF1 neurons to mediate stress-induced bone loss. Our data thus verify that the NTS is downstream of the BNST-VMH circuitry and necessary for SOM neuron-induced bone loss. Along with the increased bone density after specific inhibition of TH-positive nerve fibers in bone, our data consistently indicate that activation of BNST-VMH neural projections can induce bone loss through the sympathetic nervous system and NE.

In summary, we have uncovered a GABAergic neural circuit from the forebrain to hypothalamus that is used in the regulation of chronic stress-induced bone loss, and have also revealed a population of somatostatin neurons in the posterior region of the BNST that had not been previously implicated in bone mass 
regulation. We showed that Vglut2 neurons in the NTS and the peripheral sympathetic system were employed by this BNST-VMH neural circuit to regulate stress-induced bone loss. Our findings, therefore, not only identify the underlying central neural mechanism of stress-induced bone loss at the circuit level, but also provide a central target for therapeutic interventions in stress-induced bone metabolism disorders.

\section{Methods}

Further details on methods can be found in Supplemental Methods.

Animals. All animals were housed at $22^{\circ} \mathrm{C}-25^{\circ} \mathrm{C}$ on a circadian 12-hour light/12-hour dark cycle (lights on at 7 am and off at $7 \mathrm{pm}$ ) with ad libitum access to food and water. All animals used in this study were male, and all bone analysis was performed on mice at 6 months old, whereas 2-month-old mice were used for electrophysiological recording except in certain circumstances. SF1-Cre, GAD2ires-Cre, PV-Cre, SOM-Cre, CCK-Cre, Vglut2-Cre, TH-Cre, VGATChR2-eYFP, and OC-Cre mice (Jackson stock 012462, 010802, 008069, 013044, 011086, 016963, 008601, 014548, and 019509) were obtained from The Jackson Laboratory. SERT-Cre (Jackson stock 014554) were donated by Minmin Luo and Peng Cao of the National Institute of Biological Sciences, Beijing, China. Adrb2floxed mice (T006600) were purchased from GemPharmatech Co. Ltd. (Jiangsu, China). Adult male C57BL/6 mice (6-8 weeks) were purchased from Guangdong Medical Laboratory Animal Center (Guangzhou, China). All transgenes were used as homozygotes, except in certain circumstances. Mouse experiments were performed during the light cycle. No animals were involved in any previous studies, and they were sacrificed during the light cycle.

Human participants. Three male participants and 1 female participant (Chinese, aged 23-45 years) were selected as the 180-day Controlled Ecological Life Support System (CELSS) crew after consideration of psycho-physiological health, occupational background, age, and sex.

180-day CELSS-integrated experiment. A 180-day CELSS-integrated experiment was implemented to simulate a space residence to prepare for future deep-space exploration. It lasted for 180 days, from June 17, 2016, to December 14, 2016. The experimental facility was located in the Space Institute of Southern China, Shenzhen, China. The 180-day CELSS crew lived in this spaceship-like habitat with continuous temporal and spatial isolation, realistic mission activities, and other important special conditions of a space station. Bone mineral density was determined once before (day 0) and once after (day 180) the experiment by dual-energy x-ray absorptiometry. The Self-Rating Anxiety Scale (SAS), which focuses on the most common general anxiety disorders, was used to measure crewmember anxiety levels. Immediately before and during the mission, crewmembers filled out several computerized SAS questionnaires and rating scales. There were 20 questions, 15 pertaining to increases in crewmember anxiety levels and 5 pertaining to decreases in anxiety levels. The 4 crewmembers indicated their current anxiety status by responding to all questions using a 4-point scale from "none of the time" to "most of the time." Physical activity level was monitored on a monthly basis.

Unpredictable chronic mild stress model. C57BL/6, SOM-Cre, SERT$\mathrm{Cre}$, or OC-Cre mice were exposed to environmental stressors for 8 weeks. The following stressors were applied: (a) tight squeeze for 2 hours, where 4 mice were housed in a relatively small box $(3 \mathrm{~cm} \times 5$ $\mathrm{cm} \times 7 \mathrm{~cm}$ ); (b) wet environment for 6 hours, where water was added to home cages to moisten bedding to dampen without generating large pools; and (c) high-elevation stress, where mice were placed on a platform raised $100 \mathrm{~cm}$ above floor height for 2 hours. Stressors were randomized and counterbalanced such that each mouse received the same number of each stressor across consecutive days for the duration of 8 weeks. Efficacy of the induced-anxiety procedures was confirmed by animal behavior studies detailed in Supplemental Methods.

Statistics. Experiment data are expressed as the mean \pm SD of the number of tests stated. Statistical comparisons were made using either 2-tailed Student's $t$ test or ANOVA followed by Bonferroni's multiple-comparisons post hoc test, as indicated in the figure legends. All the statistical tests were performed using the StatView (version 10.0, SPSS) program package or Prism 7.0 software (GraphPad Software). A $P$ value of less than 0.05 was considered statistically significant.

Study approval. All animal experiments and procedures were carried out in accordance with protocols approved by the ethics committee of the Shenzhen Institutes of Advanced Technology, Chinese Academy of Sciences. The human study of this research was conducted in accordance with the principles expressed in the Declaration of Helsinki. The ethical committee of the China Astronaut Research and Training Center reviewed and approved the 180-day study, and all crewmembers gave written informed consent.

\section{Author contributions}

FY, Y. Li, and LW supervised the study and wrote the manuscript. FY and Y. Liu designed the experiments; conducted optogenetic or chemogenetic experiments, qPCR, and animal behavior experiments; and analyzed data. SC and JS performed electrophysiological recordings in brain slices. ZD and Y. Li collected and analyzed human experimental data. DY, DG, and YW conducted molecular experiments. TW, LZ, and WWL performed bone analysis. ZZ conducted trans-synaptic tracer labeling. The order of the co-first authors was assigned on the basis of their relative contributions to the study.

\section{Acknowledgments}

We are grateful to all the members of the Wang and Yang laboratories for providing comments and advice throughout the project. This project was partly supported by the following grants: National Natural Science Foundation of China (81471164, 31800881, 31630031, and 31930047); Key-Area Research and Development Program of Guangdong Province (2018B030331001); Key Research Program of Frontier Sciences of Chinese Academy of Sciences (QYZDB-SSW-SMC056); International Partnership Program of Chinese Academy of Sciences (172644KYS820170004); the Strategic Priority Research Program of Chinese Academy of Sciences (XDB32030100); Natural Science Foundation of Guangdong Province (2015A030313877); the Ten Thousand Talent Program; the Guangdong Special Support Program (2017TX04R305); CAS Key Laboratory of Brain Connectome and Manipulation (2019DP173024); Advanced Space Medico-Engineering Research Project of China (18035020103); the State Key Laboratory of Space Medicine Fundamentals and Application, China Astronaut Research and Training Center (SMFA18B02); Shenzhen Governmental Basic Research Grant (JCYJ20170413164535041, JCYJ20180507182301299); and Shenzhen Key Science and Technology Infrastructure Planning Project (ZDKJ20190204002). 
Address correspondence to: Fan Yang or Liping Wang, Shenzhen Institutes of Advanced Technology, Chinese Academy of Sciences, 1068 Xueyuan Boulevard, University Town of Shenzhen, Xili Nanshan, Shenzhen 518055, China. Phone: 86.755.2692.1910; Email: fan.yang@siat.ac.cn (FY). Phone:
86.755.8691.0600; Email:lp.wang@siat.ac.cn (LW). Or to: Yinghui Li, China Astronaut Research and Training Center, Intersection of Fengying East Road and Yongze South Road, Haidian District, Beijing 100094, China. Phone: 86.10.6636.2021; Email: yinghuidd@vip.sina.com.
1. Cheng BH, Chen PC, Yang YH, Lee CP, Huang $\mathrm{KE}$, Chen VC. Effects of depression and antidepressant medications on hip fracture: a population-based cohort study in Taiwan. Medicine (Baltimore). 2016;95(36):e4655.

2. Hong-Jhe $\mathrm{C}$, et al. The incidence and risk of osteoporosis in patients with anxiety disorder: a population-based retrospective cohort study. Medicine (Baltimore). 2016;95(38):e4912.

3. Williams LJ, et al. Depression as a risk factor for fracture in women: a 10 year longitudinal study. J Affect Disord. 2016;192:34-40.

4. Gale CR, Dennison EM, Edwards M, Sayer AA, Cooper C. Symptoms of anxiety or depression and risk of fracture in older people: the Hertfordshire Cohort Study. Arch Osteoporos. 2012;7:59-65.

5. Williams LJ, Pasco JA, Jacka FN, Hodge JM, Kotowicz MA, Berk M. Quantitative Heel Ultrasound (QUS) measures of bone quality in association with mood and anxiety disorders. JAffect Disord. 2013;146(3):395-400.

6. Zerath E. Effects of microgravity on bone and calcium homeostasis. Adv Space Res. 1998;21(89):1049-1058.

7. Smith SM, Heer MA, Shackelford LC, Sibonga JD, Ploutz-Snyder L, Zwart SR. Benefits for bone from resistance exercise and nutrition in long-duration spaceflight: evidence from biochemistry and densitometry. J Bone Miner Res. 2012;27(9):1896-1906.

8. Smith SM, Zwart SR. Nutritional biochemistry of spaceflight. Adv Clin Chem. 2008;46:87-130.

9. Vico L, et al. Cortical and trabecular bone microstructure did not recover at weight-bearing skeletal sites and progressively deteriorated at nonweight-bearing sites during the year following international space station missions. JBone Miner Res. 2017;32(10):2010-2021.

10. Wang Y, et al. During the long way to Mars: effects of 520 days of confinement (Mars500) on the assessment of affective stimuli and stage alteration in mood and plasma hormone levels. PLoS One. 2014;9(4):e87087.

11. Basner M, et al. Psychological and behavioral changes during confinement in a 520-day simulated interplanetary mission to mars. PLoS One. 2014;9(3):e93298.

12. Karsenty G, Ferron M. The contribution of bone to whole-organism physiology. Nature. 2012;481(7381):314-320.

13. Dhillon $\mathrm{H}$, et al. Leptin directly activates SF1 neurons in the $\mathrm{VMH}$, and this action by leptin is required for normal body-weight homeostasis. Neuron. 2006;49(2):191-203.

14. Takeda S, et al. Leptin regulates bone formation via the sympathetic nervous system. Cell. 2002;111(3):305-317.

15. Yadav VK, et al. A serotonin-dependent mechanism explains the leptin regulation of bone mass, appetite, and energy expenditure. Cell.
2009;138(5):976-989.

16. Wei W, et al. Orexin regulates bone remodeling via a dominant positive central action and a subordinate negative peripheral action. Cell Metab. 2014;19(6):927-940.

17. Saper CB, Lowell BB. The hypothalamus. Curr Biol. 2014;24(23):R1111-R1116.

18. Silva BA, et al. Independent hypothalamic circuits for social and predator fear. Nat Neurosci. 2013;16(12):1731-1733.

19. Lee $\mathrm{H}$, et al. Scalable control of mounting and attack by Esr1+ neurons in the ventromedial hypothalamus. Nature. 2014;509(7502):627-632.

20. Garfield AS, et al. A parabrachial-hypothalamic cholecystokinin neurocircuit controls counterregulatory responses to hypoglycemia. Cell Metab. 2014;20(6):1030-1037.

21. Oury F, et al. CREB mediates brain serotonin regulation of bone mass through its expression in ventromedial hypothalamic neurons. Genes Dev. 2010;24(20):2330-2342.

22. Zhao L, et al. Central nervous system-specific knockout of steroidogenic factor 1 results in increased anxiety-like behavior. Mol Endocrinol. 2008;22(6):1403-1415.

23. Wang L, Chen IZ, Lin D. Collateral pathways from the ventromedial hypothalamus mediate defensive behaviors. Neuron. 2015;85(6):1344-1358.

24. Idelevich A, Sato K, Nagano K, Rowe G, Gori F, Baron R. Neuronal hypothalamic regulation of body metabolism and bone density is galanin dependent. J Clin Invest. 2018;128(6):2626-2641.

25. Ducy P, et al. Leptin inhibits bone formation through a hypothalamic relay: a central control of bone mass. Cell. 2000;100(2):197-207.

26. Elefteriou F, et al. Leptin regulation of bone resorption by the sympathetic nervous system and CART. Nature. 2005;434(7032):514-520.

27. Zhao S, et al. Cell type-specific channelrhodopsin-2 transgenic mice for optogenetic dissection of neural circuitry function. Nat Methods. 2011;8(9):745-752.

28. Lebow MA, Chen A. Overshadowed by the amygdala: the bed nucleus of the stria terminalis emerges as key to psychiatric disorders. Mol Psychiatry. 2016;21(4):450-463.

29. Lindberg D, Chen P, Li C. Conditional viral tracing reveals that steroidogenic factor 1-positive neurons of the dorsomedial subdivision of the ventromedial hypothalamus project to autonomic centers of the hypothalamus and hindbrain. $J$ Comp Neurol. 2013;521(14):3167-3190.

30. Travagli RA, Anselmi L. Vagal neurocircuitry and its influence on gastric motility. Nat Rev Gastroenterol Hepatol. 2016;13(7):389-401.

31. Zoccal DB, Furuya WI, Bassi M, Colombari DS, Colombari E. The nucleus of the solitary tract and the coordination of respiratory and sympathetic activities. Front Physiol. 2014;5:238.

32. Hediger ML, England LJ, Molloy CA, Yu KF, Man-
ning-Courtney P, Mills JL. Reduced bone cortical thickness in boys with autism or autism spectrum disorder. JAutism Dev Disord. 2008;38(5):848-856.

33. Gale CR, Dennison EM, Edwards M, Sayer AA, Cooper C. Symptoms of anxiety or depression and risk of fracture in older people: the Hertfordshire Cohort Study. Arch Osteoporos. 2012;7:59-65.

34. Heikkinen J, et al. Depressive disorders, anxiety disorders and subjective mental health in common musculoskeletal diseases: a review. Maturitas. 2019;127:18-25.

35. Sato S, et al. Central control of bone remodeling by neuromedin U. Nat Med. 2007;13(10):1234-1240.

36. Herber CB, et al. Estrogen signaling in arcuate Kiss1 neurons suppresses a sex-dependent female circuit promoting dense strong bones. Nat Commun. 2019;10(1):163.

37. Kim JG, et al. AgRP neurons regulate bone mass. Cell Rep. 2015;13(1):8-14.

38. Choi YH, Fujikawa T, Lee J, Reuter A, Kim KW. Revisiting the ventral medial nucleus of the hypothalamus: the roles of SF-1 neurons in energy homeostasis. Front Neurosci. 2013;7:71.

39. Grayson BE, Seeley RJ, Sandoval DA. Wired on sugar: the role of the CNS in the regulation of glucose homeostasis. Nat Rev Neurosci. 2013;14(1):24-37.

40. Mobbs CV, Moreno CL, Poplawski M. Metabolic mystery: aging, obesity, diabetes, and the ventromedial hypothalamus. Trends Endocrinol Metab. 2013;24(10):488-494.

41. Flak JN, et al. Leptin-inhibited PBN neurons enhance responses to hypoglycemia in negative energy balance. Nat Neurosci. 2014;17(12):1744-1750.

42. Kim KW, Zhao L, Parker KL. Central nervous system-specific knockout of steroidogenic factor 1. Mol Cell Endocrinol. 2009;300(1-2):132-136.

43. da Silva ES, Poltronieri SC, Nascimento JO, Zangrossi H, Viana MB. Facilitation of 5-HT(2A/2C)-mediated neurotransmission in the ventromedial hypothalamic nucleus decreases anxiety in the elevated T-maze. Behav Brain Res. 2011;216(2):692-698.

44. Yang CF, et al. Sexually dimorphic neurons in the ventromedial hypothalamus govern mating in both sexes and aggression in males. Cell. 2013;153(4):896-909.

45. Musatov S, Chen W, Pfaff DW, Kaplitt MG, Ogawa S. RNAi-mediated silencing of estrogen receptor $\alpha$ in the ventromedial nucleus of hypothalamus abolishes female sexual behaviors. Proc Natl Acad Sci U S A. 2006;103(27):10456-10460.

46. Frankfurt M, Fuchs E, Wuttke W. Sex differences in gamma-aminobutyric acid and glutamate concentrations in discrete rat brain nuclei. Neurosci Lett. 1984;50(1-3):245-250.

47. Sparta DR, Jennings JH, Ung RL, Stuber GD. Optogenetic strategies to investigate neural circuitry engaged by stress. Behav Brain Res. 
2013;255:19-25.

48. Pompolo S, Ischenko O, Pereira A, Iqbal J, Clarke IJ. Evidence that projections from the bed nucleus of the stria terminalis and from the lateral and medial regions of the preoptic area provide input to gonadotropin releasing hormone (GNRH) neurons in the female sheep brain. Neuroscience. 2005;132(2):421-436.

49. Zhang W, Zhang N, Sakurai T, Kuwaki T. Orexin neurons in the hypothalamus mediate cardiorespiratory responses induced by disinhibition of the amygdala and bed nucleus of the stria terminalis. Brain Res. 2009;1262:25-37.

50. Jennings JH, Rizzi G, Stamatakis AM, Ung RL, Stuber GD. The inhibitory circuit architecture of the lateral hypothalamus orchestrates feeding. Science. 2013;341(6153):1517-1521.

51. Radley JJ, Gosselink KL, Sawchenko PE. A discrete GABAergic relay mediates medial prefrontal cortical inhibition of the neuroendocrine stress response. J Neurosci. 2009;29(22):7330-7340.
52. Chan O, et al. Increased GABAergic output in the ventromedial hypothalamus contributes to impaired hypoglycemic counterregulation in diabetic rats. Diabetes. 2011;60(5):1582-1589.

53. Moore AM, Prescott M, Marshall CJ, Yip SH, Campbell RE. Enhancement of a robust arcuate GABAergic input to gonadotropin-releasing hormone neurons in a model of polycystic ovarian syndrome. Proc Natl Acad Sci U S A. 2015;112(2):596-601.

54. Kepecs A, Fishell G. Interneuron cell types are fit to function. Nature. 2014;505(7483):318-326.

55. Ahrens S, et al. A central extended amygdala circuit that modulates anxiety. J Neurosci. 2018;38(24):5567-5583.

56. Lin LC, Sibille E. Somatostatin, neuronal vulnerability and behavioral emotionality. Mol Psychiatry. 2015;20(3):377-387.

57. Luo SX, et al. Regulation of feeding by somatostatin neurons in the tuberal nucleus. Science. 2018;361(6397):76-81.
58. Campbell JN, et al. A molecular census of arcuate hypothalamus and median eminence cell types. Nat Neurosci. 2017;20(3):484-496.

59. Lucas D, et al. Chemotherapy-induced bone marrow nerve injury impairs hematopoietic regeneration. Nat Med.2013;19(6):695-703.

60. Mantyh PW. The neurobiology of skeletal pain. Eur JNeurosci. 2014;39(3):508-519.

61. Kajimura D, et al. Genetic determination of the cellular basis of the sympathetic regulation of bone mass accrual. JExp Med.2011;208(4):841-851.

62. Nagao M, et al. Sympathetic control of bone mass regulated by osteopontin. Proc Natl Acad Sci US A. 2011;108(43):17767-17772.

63. Fukuda T, et al. Sema3A regulates bone-mass accrual through sensory innervations. Nature. 2013;497(7450):490-493.

64. Zhang Y, et al. Implant-derived magnesium induces local neuronal production of CGRP to improve bone-fracture healing in rats. Nat Med. 2016;22(10):1160-1169. 